\title{
Physical Reflectivity and Polarization Characteristics for Snow and Ice-Covered Surfaces Interacting with GPS Signals
}

\author{
Nasser Najibi ${ }^{1,2, *}$ and Shuanggen Jin ${ }^{1}$ \\ 1 Shanghai Astronomical Observatory, Chinese Academy of Sciences, Shanghai 200030, China; \\ E-Mail: sgjin@shao.ac.cn \\ 2 University of Chinese Academy of Sciences, Beijing 100049, China \\ * Author to whom correspondence should be addressed; E-Mail: nsr.najibi@gmail.com; \\ Tel.: +86-21-3477-5292; Fax: +86-21-6438-4618.
}

Received: 31 May 2013; in revised form: 22 July 2013 / Accepted: 23 July 2013 /

Published: 9 August 2013

\begin{abstract}
The Global Positioning System (GPS) reflected signal has been demonstrated to remotely sense the oceans, land surfaces and the cryosphere, including measuring snow depth, soil moisture, vegetation growth and wind direction. Since the Earth surface's characteristics are very complex, the surface reflectivity process and interaction with GPS signals is not well understood. In this study, we investigate the surface's reflectivity and variability of snow and ice surfaces interacting with GPS L1 and L2 signals in order to retrieve multipath signals and infer surface characteristics by using the direct and reflected polarizations of each signal. Firstly, the effects of both GPS satellite elevation angle and GPS receiver's antenna height variations on the multipath signal variability have been investigated by numerical formulations. Secondly, the specular reflection coefficients' features and the total surface polarization for liquid and solid surfaces are discussed. Moreover, the linear polarization and circular polarizations (co-polarized and cross-polarized) as well as their corresponding convolution functions are developed horizontally and vertically. The results show that the multipath signals are more sensitive to the satellite elevation angle variations than to changes in the GPS receiver's antenna height. The convolution function demonstrates that the snowy surface has a minimum reflectance in circular polarization but maximum reflectance in linear polarization. GPS signals reflecting from an ice-covered surface show a maximum value in circular polarization reflectance and a minimum for linear polarization reflectance. Moreover, the values for reflection from soils are between those for snow and ice in all polarization types. The placement of soil surface reflectance values between snowy and icy surface ones may be noteworthy in new remote sensing applications.
\end{abstract}


Keywords: snow; ice; reflectivity; polarization; GPS antenna height; satellite elevation angle; convolution function

\section{Introduction}

Nowadays, GPS reflectometry and scatterometry have incredible applications in the field of the Earth system sciences as new remote sensing tools in oceanography, hydrology and glaciology $[1,2]$. GPS reflectometry provides a way to infer the surface properties remotely because the reflectance spectra at radio wavelengths contain properties about the surface compositions [3-5]. The high reflectivity of GPS signals in the frequency range of L-Band $(1.2276$ and $1.5754 \mathrm{GHz})$ on the liquid water and the frozen water (e.g., ice caps, glaciers, etc.) allows us to detect the reflected signals reasonably well [6].

Many studies have been done to observe surface reflectance features. For example, Hannah [7] developed several multipath propagation simulations for concrete, dry ground, medium dry ground, wet ground, fresh water and sea water in linear and circular and also effective polarizations. This study did not consider snowy and icy surfaces or the physical reflectivity of scattered and reflected signals on its simulations and results.

Recently, Litvinov et al. [8] investigated the reflection properties of soil and vegetation surfaces by using airborne data to model the total and polarized reflectances in the unique form for both soil and vegetation surfaces. They performed their experiments with airborne data instead of ground GPS reflected signals. Moreover, Chew et al. [9] demonstrated the influence of soil moisture variations for bare soil conditions on both amplitude and phase of multipath signature using Signal to Noise Ratio (SNR) simulated data.

To retrieve the surface properties from ground GPS signals, the physical surface reflectivity of the scattered and reflected signals should be taken into account. Additionally, the chemical surface characteristic for different reflectance polarizations and different grazing angles in interacting with the GPS signals must be understood in detail. Elosegui et al. [10] introduced the GPS multipath signals for different satellite elevation angles with a constant GPS antenna height and a certain ratio of GPS reflected amplitude signals, which proved that GPS multipath signature is a function of GPS antenna height, ratio of reflected GPS signal, satellite elevation angle and the wavelength. However, they did not discuss the variability of GPS multipath signature affected by the changes in the GPS antenna height or satellite elevation angles variations.

In this paper, the effects of integrated satellite elevation angles variations and GPS antenna height's changes on multipath signature's variability are investigated by analyzing numerically the phase shift variation formula of reflected signals in comparison to the combined signals (direct and reflected signals). Then, multipath signature variability during the critical conditions including GPS scattered and reflected signals in the satellite zenith angle and the minimum or maximum GPS antenna height are discussed.

In addition, the reaction of snow, ice and soil surface to GPS scattered L1 and L2 signals, characteristically including the specular reflection, total surface reflectivity, the linear polarization and 
circular polarization for both GPS L1 and L2 signals, are analyzed and their corresponding convolution functions are discussed in detail for snowy and icy surfaces. Snow and ice-covered surfaces indeed play important roles in environmental systems, especially hydrologic cycles and climate change, and thus, it is worthwhile to focus on them.

\section{Theory and Methodology}

As we can see in Figure 1, two types of polarized signals from GPS satellite and also existent surfaces can be acquired by ground GPS receiver: Right-Hand Circular Polarization (RHCP) and Left-Hand Circular Polarization (LHCP). It should be noted that the reflected surface's signal is not only LHCP, but in fact, it is a combination of RHCP and LHCP signals. Generally, incident RHCP for the angles with less than the Brewster angle produces mainly a RHCP reflected wave. Incident RHCP for the angles with greater than the Brewster angle produces a LHCP reflected wave. At the Brewster angle, the reflected signal is linearly polarized (Figure 1).

Figure 1. General surface reflectance process in GPS L1 and L2 signals for Right-Hand Circular Polarization (RHCP) and Left-Hand Circular Polarization (LHCP) components and the Brewster angle (not to scale).

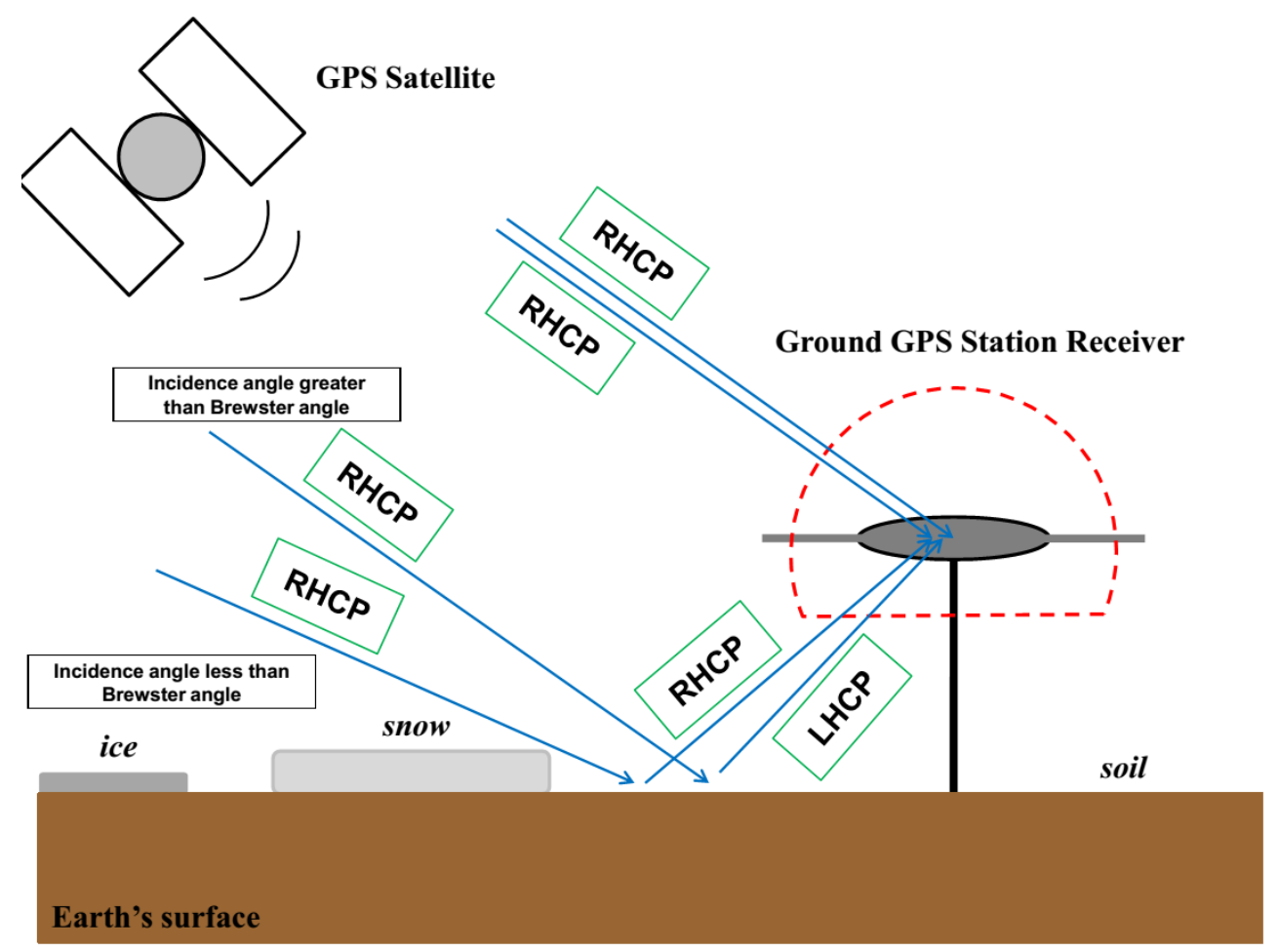

In order to sense and discriminate the snow surface and ice surface using ground GPS receivers' observations, the physical geometry of GPS reflected signals and the characteristics of surface reflected signals must be analyzed. This can be done through investigation of the physical status of both scattered and reflected signals as well as the polarization of the targeted surface in different propagation angles. The general flowchart of the proposed methodology to sense snowy and icy surfaces is given in Figure 2. 
The general procedure is described as follows: Firstly, the physical reflectivity of GPS for both scattered and reflected signals is considered in the presence of time variability for GPS satellite elevation angles and GPS antenna height. Meanwhile, the reflectance characteristic is investigated with polarization in snowy and icy surfaces by linear and circular polarization processes. These polarizations are considered as inputs for convolution function in order to distinguish the difference in snowy and icy surfaces (Figure 2).

Figure 2. Flowchart of the proposed methodology to sense snowy and icy surfaces via GPS reflected signals using ground GPS station receivers' observations.

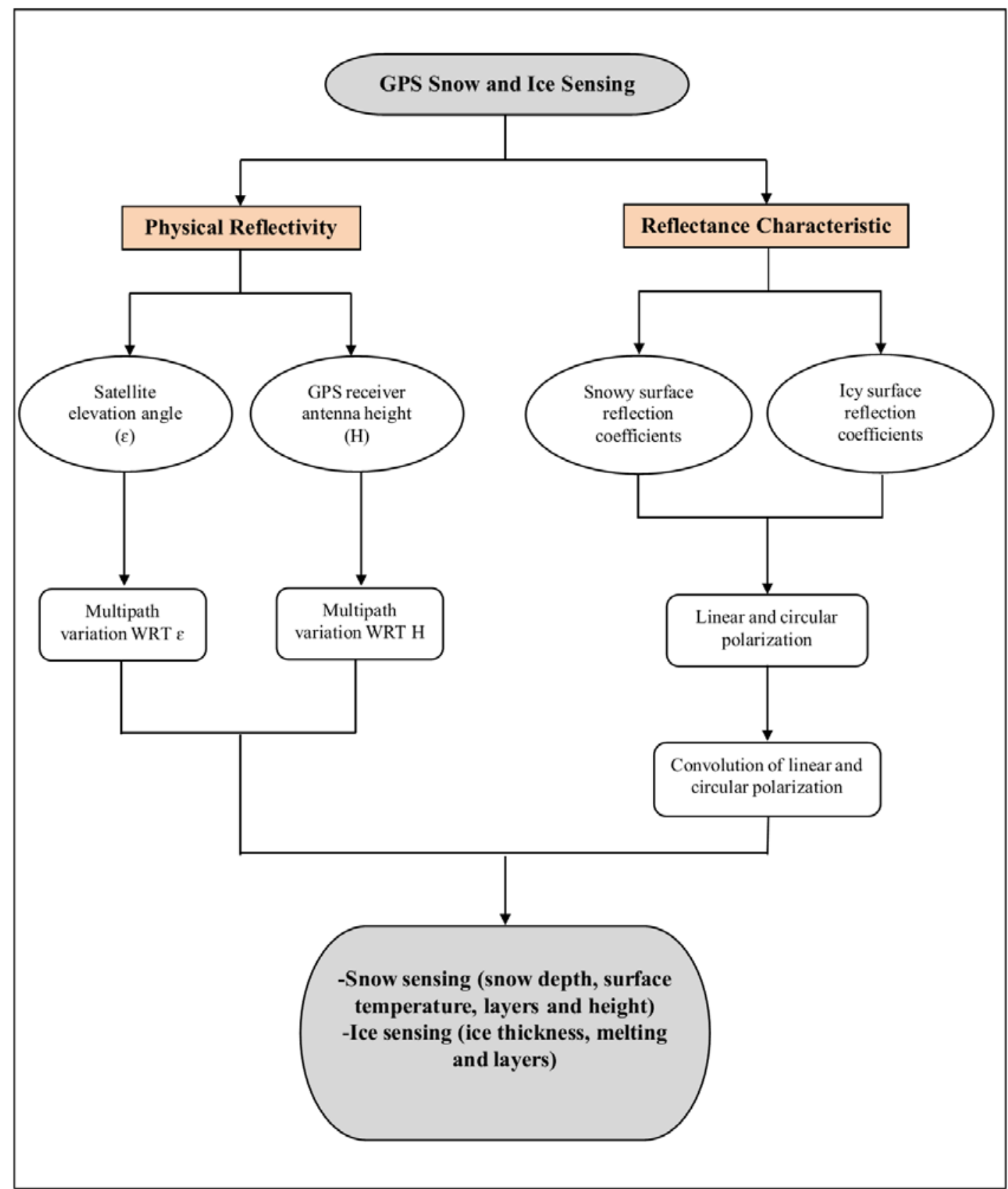

In both cases, we generally deal with GPS scattered and reflected signals; but in physical reflectivity, we ignore the chemical characteristics of the surface and thus, we only concentrate on the surface's physical status, such as the variations related to its physical shape rather than the material features (and so we treat snow and ice surfaces as a unique surface). However, in reflectance 
characteristic procedures, we pay attention only to the chemical properties of the surface, such as conductivity, relative permittivity and polarization parameters.

The physical outer surface of snow, ice and ground (soil) is initially assumed as flat and parallel to the local horizontal plane (perpendicular to the local plumb line) and the influence of undesired external factors do not exist, such as any sudden fluctuations in the satellite elevation angle or replacement of the GPS antenna,. Additionally, any irregularity in density and temperature of these materials that will result in inhomogeneous values for conductivity and relative permittivity are negligible.

In the following, physical reflectivity and reflectance characteristics' steps are discussed in detail, respectively.

\subsection{Physical Reflectivity}

Multipath signature depends on the following four parameters: the GPS wavelength $(\lambda)$, ratio of the reflected wave amplitude relative to the direct wave $(\alpha)$, GPS satellite elevation angle $(\varepsilon)$, and the GPS antenna height $(H)$. It can be written as $[10,11]$ :

$$
\delta \Phi=\tan ^{-1}\left(\frac{\alpha \sin \left(4 \pi \frac{H}{\lambda} \sin \varepsilon\right)}{1+\alpha \cos \left(4 \pi \frac{H}{\lambda} \sin \varepsilon\right)}\right)=\delta \Phi(\lambda, \alpha, \varepsilon, H)
$$

where $\delta \Phi$ is the reflected wave phase shift value related to the total (the reflected wave plus a direct one) (see Figure 3).

Figure 3. GPS scattered and reflected signals towards the Earth's surface and from surrounding ground GPS station receiver during a specified time span while the $H$ is constant.

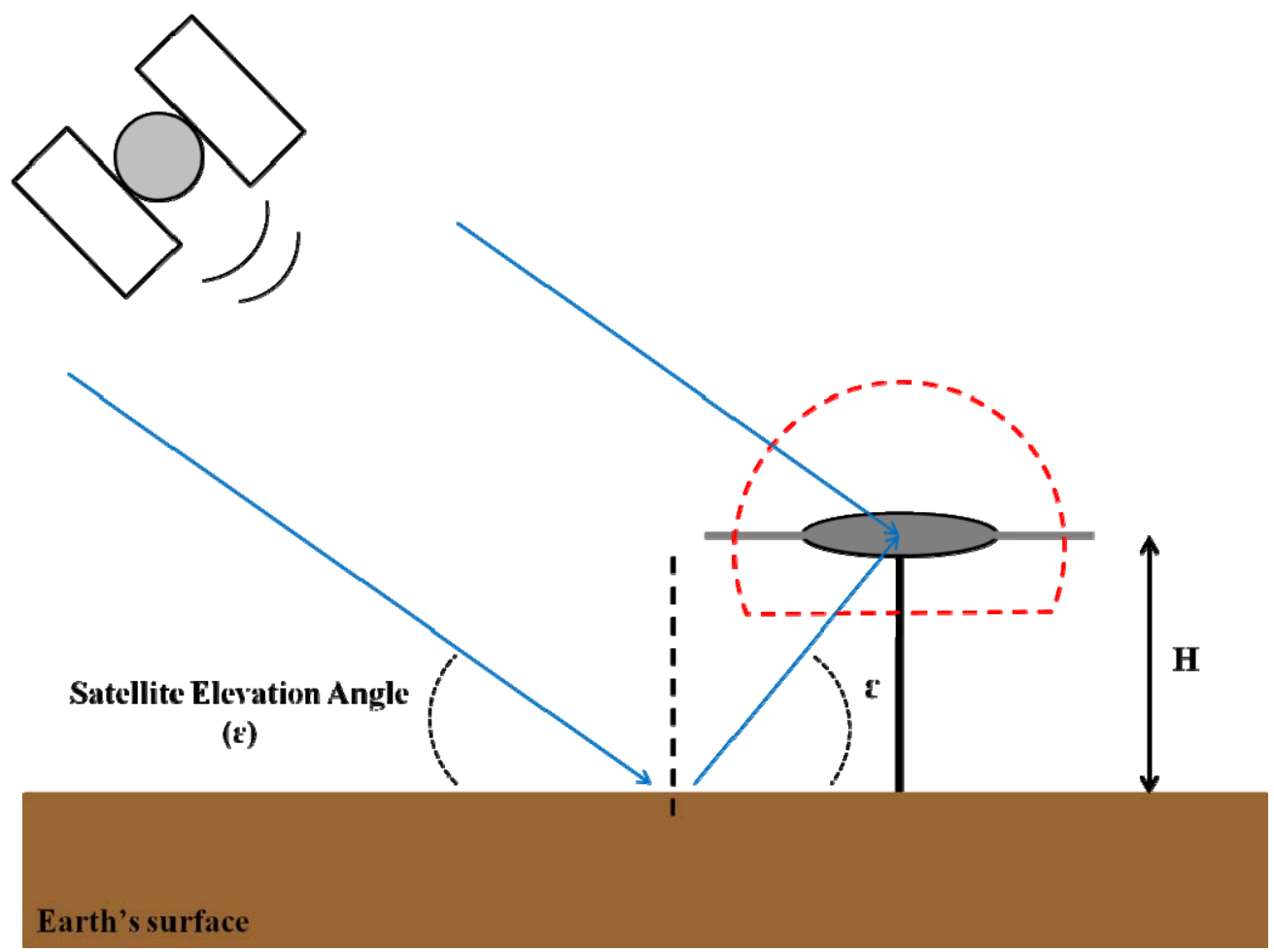


Referring to Equation (1), we analyze two conditions, including multipath variations with respect to GPS satellite elevation variability $(d \varepsilon)$ and multipath variations with respect to GPS antenna height variability $(d H)$ with the assumption that $\alpha$ is a constant parameter.

\subsubsection{Multipath Variations with Respect to Satellite Elevation Angle Variability $(d \varepsilon)$}

If we rewrite Equation (1) with the following substitution:

$$
M=4 \pi \frac{H}{\lambda}
$$

Therefore:

$$
\delta \Phi=\tan ^{-1}\left(\frac{\alpha \sin (M \sin \varepsilon)}{1+\alpha \cos (M \sin \varepsilon)}\right)=\delta \Phi(\alpha, M, \varepsilon)
$$

Moreover, if we assume a certain condition whereby the GPS antenna height $(H)$ does not change, $M$ will not vary during this certain time period. Therefore, the changes in multipath during the time (velocity of multipath signature $\left(V_{\delta \Phi}\right)$ ) related to the changes in the satellite elevation angle $(\varepsilon)$ (velocity of satellite elevation angle $\left(V_{\varepsilon}\right)$ ) can be defined as:

$$
\frac{V_{\delta \Phi}}{V_{\varepsilon}}=\frac{d \delta \Phi / d t}{d \varepsilon / d t}=\frac{d \delta \Phi}{d \varepsilon}=\frac{\frac{d}{d \varepsilon}\left(\frac{\alpha \sin (M \sin \varepsilon)}{1+\alpha \cos (M \sin \varepsilon)}\right)}{1+\left(\frac{\alpha \sin (M \sin \varepsilon)}{1+\alpha \cos (M \sin \varepsilon)}\right)^{2}}
$$

and simplifying Equation (4) gives:

$$
\frac{V_{\delta \Phi}}{V_{\varepsilon}}=\frac{d \delta \Phi}{d \varepsilon}=M \alpha \frac{\alpha \cos \varepsilon+\cos \varepsilon \cos (M \sin \varepsilon)}{(1+\alpha \cos (M \sin \varepsilon))^{2}}
$$

Equation (5) shows that the function of multipath signature variations based on satellite elevation angle's changes significantly depends on $H$ value embedded in $M$, which is a function of $H$ straightforwardly (Equation (2)).

Moreover, multipath variations with respect to the satellite elevation angle variability for two critical conditions depend on not only the satellite elevation angle but also GPS antenna height. For instance, at the certain point that GPS satellite elevation angle is equal to zero, it is given as:

$$
\varepsilon=0 \rightarrow \frac{d \delta \Phi}{d \varepsilon}=M \alpha \frac{\alpha+1}{(1+\alpha)^{2}}=\frac{M \alpha}{1+\alpha}
$$

It is clear from Equation (6) that the multipath signature variability with respect to the changes in satellite elevation angle interrelates directly to GPS antenna height at the certain point when the satellite elevation angle is zero. However, the multipath signature variability with respect to the changes in the satellite elevation angle in the GPS satellite zenith angle (when GPS satellite elevation angle is $\pi / 2$ ) will be concluded as:

$$
\varepsilon=\frac{\pi}{2} \rightarrow \frac{d \delta \Phi}{d \varepsilon}=0
$$


Equation (7) describes when the GPS satellite is directly, vertically placed above the ground GPS receiver antenna; the variability of multipath signature with respect to the $\varepsilon$ variations is nullification.

\subsubsection{Multipath Variations with Respect to GPS Antenna Height Variability $(d H)$}

In a similar fashion, the multipath signature's variability with respect to the changes in the GPS antenna height during the specific time span being computed, it can be written as:

$$
\frac{V_{\delta \Phi}}{V_{H}}=\frac{d \delta \Phi / d t}{d H / d t}=\frac{d \delta \Phi}{d H}=\frac{\frac{d}{d H}\left(\frac{\alpha \sin (N H)}{1+\alpha \cos (N H)}\right)}{1+\left(\frac{\alpha \sin (N H)}{1+\alpha \cos (N H)}\right)^{2}}
$$

where the variables are the same ones described in Equation (1) and $N$ is defined as a function of satellite elevation angle $(\varepsilon)$, as follows:

$$
N=\frac{4 \pi}{\lambda} \sin \varepsilon
$$

Simplifying Equation (8) can give us the following uncomplicated relationship between multipath signature's velocity and $H$, as:

$$
\frac{V_{\delta \Phi}}{V_{H}}=\frac{d \delta \Phi}{d H}=N \alpha \frac{(\cos (N H)+\alpha)}{(\alpha+\cos (N H))^{2}+\sin ^{2}(N H)}
$$

In a similar way, if the GPS antenna height becomes zero $(H=0)$, then:

$$
\frac{d \delta \Phi}{d H}=N \frac{\alpha}{1+\alpha}
$$

By referring to Equation (9), Equation (11) demonstrates how important the satellite elevation angle is in multipath signatures variability's analysis when the GPS antenna heights are simultaneously changing.

In order to understand when the multipath signatures variability is going to be zero (in the situation that the multipath signature variability is independent from the GPS antenna height variations) with respect to the changes in GPS antenna height; therefore:

$$
\frac{d \delta \Phi}{d H}=0 \rightarrow N \alpha(\cos (N H)+\alpha)=0 \rightarrow\left\{\begin{array}{l}
\varepsilon=\sin ^{-1}\left(\frac{\lambda}{8 H}\right) \\
\varepsilon=\sin ^{-1}\left(\frac{3 \lambda}{16 H}\right)
\end{array}\right.
$$

Included in Equation (12) are two important assumptions as $\cos ^{-1}(-\alpha) \approx \pi / 2(\alpha=0.06$ from Elosegui et al. [10]) and also satellite elevation angle cannot be equaled to zero value during that specific time span. In the other words, if the satellite elevation angle may be equated to one of the given values for $\varepsilon$ in Equation (12) $\left(\sin ^{-1}(\lambda / 8 H)\right.$ or $\left.\sin ^{-1}(3 \lambda / 16 H)\right)$, the multipath signatures' variability will be independent of any changes in the GPS antenna height.

\subsection{Reflectance Characteristic}

Generally, the polarization of an electromagnetic wave is defined as the orientation of the electric field vector where the electric field vector is perpendicular to both the direction of travel and the 
magnetic field vector [12]. The polarization is introduced as the geometric figure projected by the electrical field vector in a static plane perpendicular to the direction of propagation while the wave passes through that assumed plane [13]. In fact, when the radio waves are transmitting from a material or space with given refractive index $\mathrm{n}_{1}$ into a second material or space with refractive index $\mathrm{n}_{2}$, both reflection and refraction of the waves may occur [14-16].

In spectroscopy, the Fresnel equations identify which wave fraction is reflected and which one is refracted (i.e., transmitted). Furthermore, they describe the phase shift related to the reflected wave [7]. Generally in reflectance characteristics, we are dealing with the surface chemical property constants, especially conductivity and relative permittivity. The conductivity and relative permittivity values for soil, snow and ice surfaces used in this study are given in Table 1 ([17-19]).

Table 1. Conductivity and relative permittivity properties values [17-19].

\begin{tabular}{ccc}
\hline Surface & Conductivity $(\mathbf{m h o} / \mathbf{m})$ & Relative Permittivity $\left(\varepsilon_{r}\right)$ \\
\hline soil & $1 \times 10^{-5}$ & 4 to 8 \\
snow & $10^{-9}$ to $10^{-7}$ & 4 \\
ice & $1 \times 10^{-5}$ to $3 \times 10^{-5}$ & 3 to 30 \\
\hline
\end{tabular}

Here the soil is considered as dry bare soil with estimated relative permittivity less than eight percent [19]. Moreover, the modeled snow is a soft new snow with density of $127 \mathrm{~kg} / \mathrm{m}^{3}$ approximately [17] and the ice is pure ice with mean temperature between 0 and $-10{ }^{\circ} \mathrm{C}$ [18]. The conductivity values for modeled soil, snow and ice surface are considered as mean values of the represented values in Table 1 .

In the surface reflectance domain, commonly two waves are being analyzed: s-polarized $\left(R_{S}\right)$ and p-polarized $\left(R_{p}\right)$. The incident wave, with an electric field located in the same plane of the interface (perpendicular to the plane of the diagram in Figure 1), is called s-polarized $\left(R_{s}\right)$. That incident wave with its electric field is placed in a perpendicular direction to the s-polarized wave (in the same geometric plane of the diagram in Figure 1) and is called p-polarized $\left(R_{p}\right)$.

\subsubsection{Surface Reflectance Characteristic for Linear and Circular Polarization}

When an electromagnetic wave is traveling from a dense medium into a less dense one (i.e., $\mathrm{n}_{1}>\mathrm{n}_{2}$ ), for a specific material those angles, which are more than the incidence angle, are known as the critical angles for that material. Then, the entire wave is being reflected and thus, $R_{s}=R_{p}=1$. This phenomenon is defined as the total internal reflection [14].

It is well-known that the reflection coefficients $(R C)$ for horizontal $\left(R C_{H}\right)$ and vertical $\left(R C_{V}\right)$ polarization are as Equations (13) and (14), where $\varepsilon=\varepsilon_{r}-j \frac{\sigma}{\omega \varepsilon_{0}}[20]$ :

$$
\begin{gathered}
R C_{H}=\frac{\sin \theta-\sqrt{\varepsilon-(\cos \theta)^{2}}}{\sin \theta+\sqrt{\varepsilon-(\cos \theta)^{2}}} \\
R C_{V}=\frac{\varepsilon \sin \theta-\sqrt{\varepsilon-(\cos \theta)^{2}}}{\varepsilon \sin \theta+\sqrt{\varepsilon-(\cos \theta)^{2}}}
\end{gathered}
$$


where $\varepsilon$ is the complex dielectric constant with assumption of time dependence in $e^{-j \omega t}[13,21]$ and $\theta$ is the grazing angle.

By substituting for $\omega$ and $\varepsilon_{0}$ in $\varepsilon$ function, it can be written as $\varepsilon=\varepsilon_{r}-j 60 \lambda \sigma$. Therefore, the calculation of each linear coefficient is now straightforward using a given frequency (e.g., GPS L1 and L2 frequency values) together with known grazing angle $(\theta)$, dielectric constant and conduction value for the reflecting surface [22].

According to GPS receiver antenna property, which only can receive RHCP signals (in fact the amount of LHCP signals which can be received by GPS receiver antenna are negligible), it is necessary to assess these selected surfaces in cross-polarized and co-polarized cases [23]. According to Leroux et al. [24], the co-polarization equation can be written as [24]:

$$
\Gamma_{O}=\frac{R C_{H}+R C_{V}}{2}
$$

Similarly for cross-polarization:

$$
\Gamma_{X}=\frac{R C_{H}-R C_{V}}{2}
$$

where $\Gamma o$ and $\Gamma_{X}$ are the reflection coefficients for co-polarization and cross-polarization and $R C_{H}$ and $R C_{V}$ are the reflection coefficients for horizontal and vertical linear polarization respectively derived from Equations (13) and (14). By using GPS L1 and L2 signal as the scatter signals towards the selected surfaces, reflection coefficients for GPS L1 and L2 signals versus grazing angles can be computed.

\subsubsection{Convolution Functions for Surface Reflectance Characteristics}

In order to get the total surface reflectance from GPS signals, the cross-correlation between vertical and horizontal components of each L1 and L2 signal is proposed since a reflected signal is correlated horizontally and vertically. The cross-correlation equation is the convolution of $R C_{H}$ and $R C_{V}$ written as $R C_{H} \times R C_{V}$. It is defined as the integral of the product of the two functions after one is reversed and shifted. This particular integral transformation is given by:

$$
\begin{aligned}
& \left(R C_{H} * R C_{V}\right)(t)=\int_{-\infty}^{+\infty} R C_{H}(\tau) * R C_{V}(t-\tau) d \tau \\
& =\int_{-\infty}^{+\infty} R C_{H}(t-\tau) * R C_{V}(\tau) d \tau
\end{aligned}
$$

where $\tau$ is a free variable and $t$ does not represent the time domain necessarily (related to each embedded convolution function in Equation (17), and $t$ can be interpreted as a random variable, independent variable or time span) [25]. Therefore, in the half plane surface angles domain (from 0 to 180 degrees), which is considered as the total angles for vertical and horizontal components, the tested surfaces have different convolution values for linear polarization in interacting with GPS L1 and L2 signals. We investigate both GPS signals in linear polarization convolution for snow, ice and soil surfaces.

Similarly the cross-correlation polarization between co-polarized and cross-polarized of each L1 and L2 signal is happening in the convolution function of both polarizations. Since the entire reflected signal surface is correlated, the cross-correlation equation is the convolution of $\Gamma_{o}$ and $\Gamma_{X}$ that is written as $\Gamma_{o} \times \Gamma_{X}$. It is defined as the integral of the product of the two functions after one is reversed and shifted $[23,25]$. This particular integral transformation is given by: 


$$
\begin{aligned}
& \left(\Gamma_{O} * \Gamma_{X}\right)(t)=\int_{-\infty}^{+\infty} \Gamma_{O}(\tau) * \Gamma_{X}(t-\tau) d \tau \\
& =\int_{-\infty}^{+\infty} \Gamma_{O}(t-\tau) * \Gamma_{X}(\tau) d \tau
\end{aligned}
$$

where $\tau$ is a free variable and $t$ can be interpreted as a random variable, independent variable or timing span. The selected surfaces for the half plane surface angles domain have different convolution function values for circular polarization in dealing with GPS L1 and L2 signals.

\section{Results and Discussion}

\subsection{GPS Reflected Signals and Physical Reflectivity}

According to Equation (1), the multipath signatures caused by GPS reflected signals are critically dependant on the satellite elevation angle $(\varepsilon)$ and GPS antenna height $(H)$. It is assumed that GPS scattered and reflected wavelengths $(\lambda)$ as well as the ratio of the reflected signal amplitude relative to the direct one $(\alpha)(\alpha=0.06$; according to [10]) are constants.

In order to present the multipath signature variability with respect to $H$ and $\varepsilon$ variations during the specified time period, the velocity equations are used. In fact, the velocity equations are the derivatives of the original shifted phase signal to the total value of direct and reflected signal upon subjecting to $H$ and $\varepsilon$ values, which have been proposed respectively in Equations (5) and (10).

Figure 4. GPS scattered and reflected signals towards the Earth's surface and from surrounding ground GPS station receiver during a specified time span while the $H$ is constant.

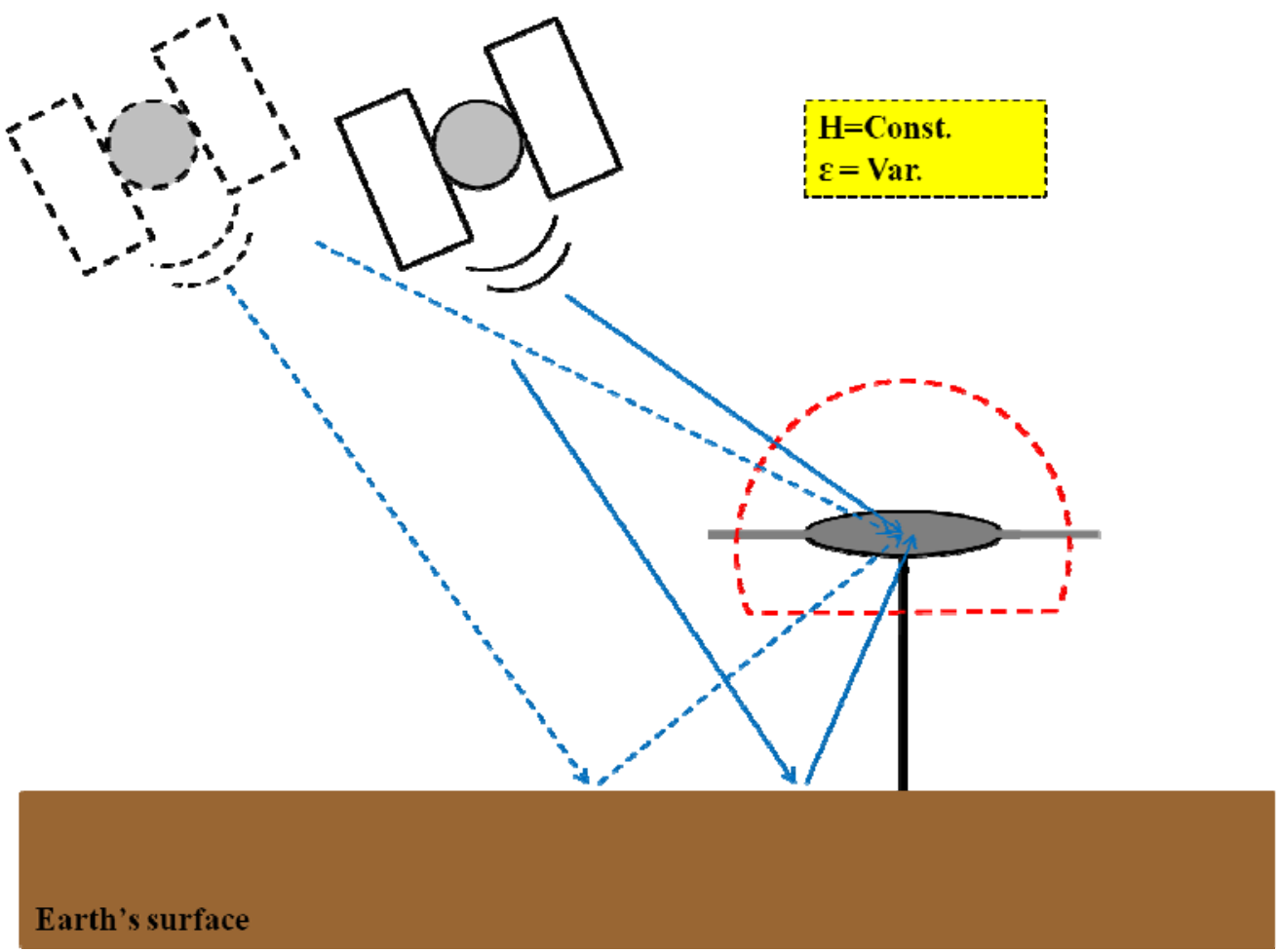


If no change has been made into surrounding the ground GPS station receiver's initial natural condition practically (e.g., snow accumulation) or the GPS antenna is not being replaced with another one, $\mathrm{H}$ will be constant (Figure 4). Thereafter, the variability of multipath signatures subjected to the changes in satellite elevation angle itself $\left(V_{\varepsilon}\right)$ and also the variability of multipath signatures subjected to the GPS antenna height variations $\left(V_{H}\right)$ can be analyzed in the different $H$ values (depending on the type of GPS antenna product). They are presented in Figures 5 and 6 , respectively.

Figure 5. Variability of multipath signatures subjected to the changes in $H\left(V_{\Phi} / V_{H}\right)$ based on $\varepsilon$ variations.

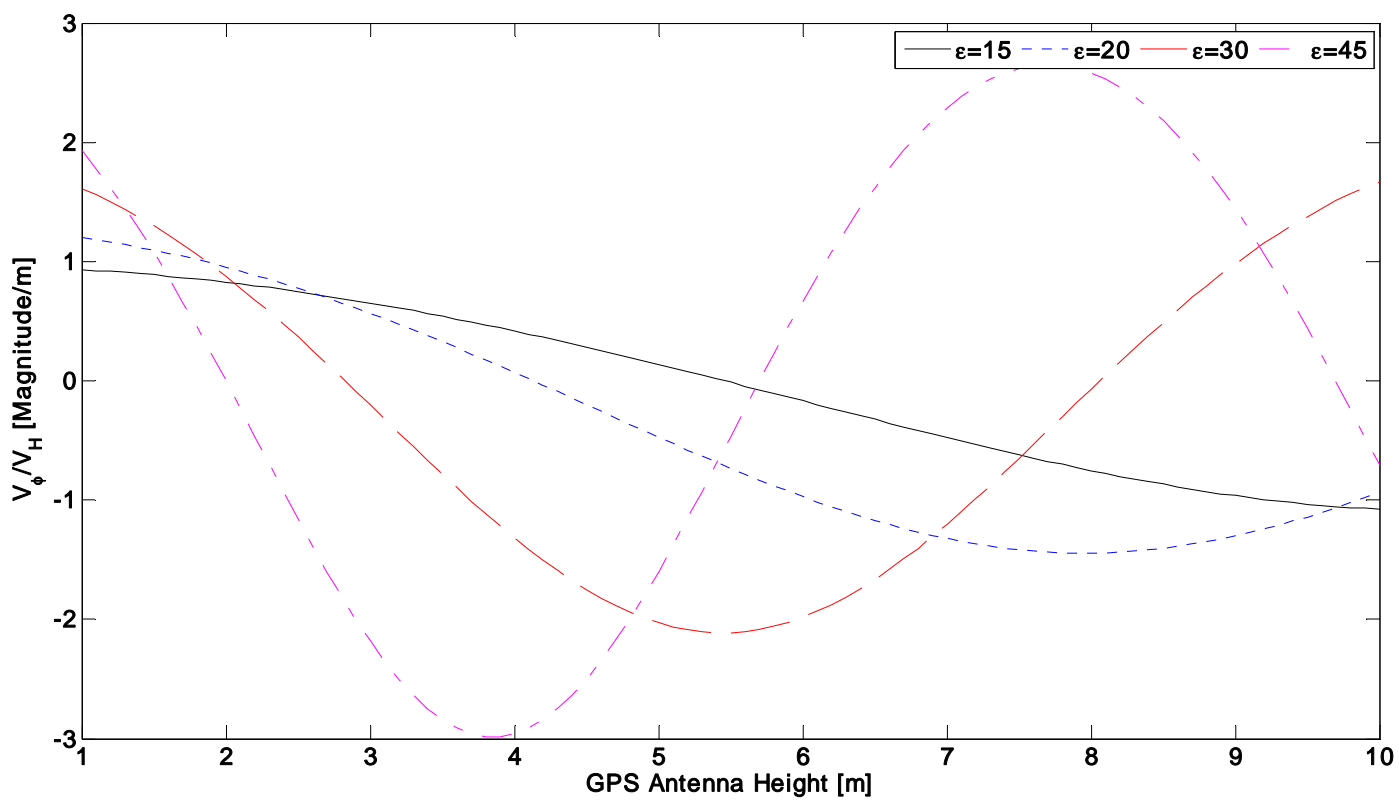

Figure 6. Variability of multipath signatures subjected to the changes in $\varepsilon\left(V_{\Phi} / V_{\varepsilon}\right)$ based on $\varepsilon$ variations.

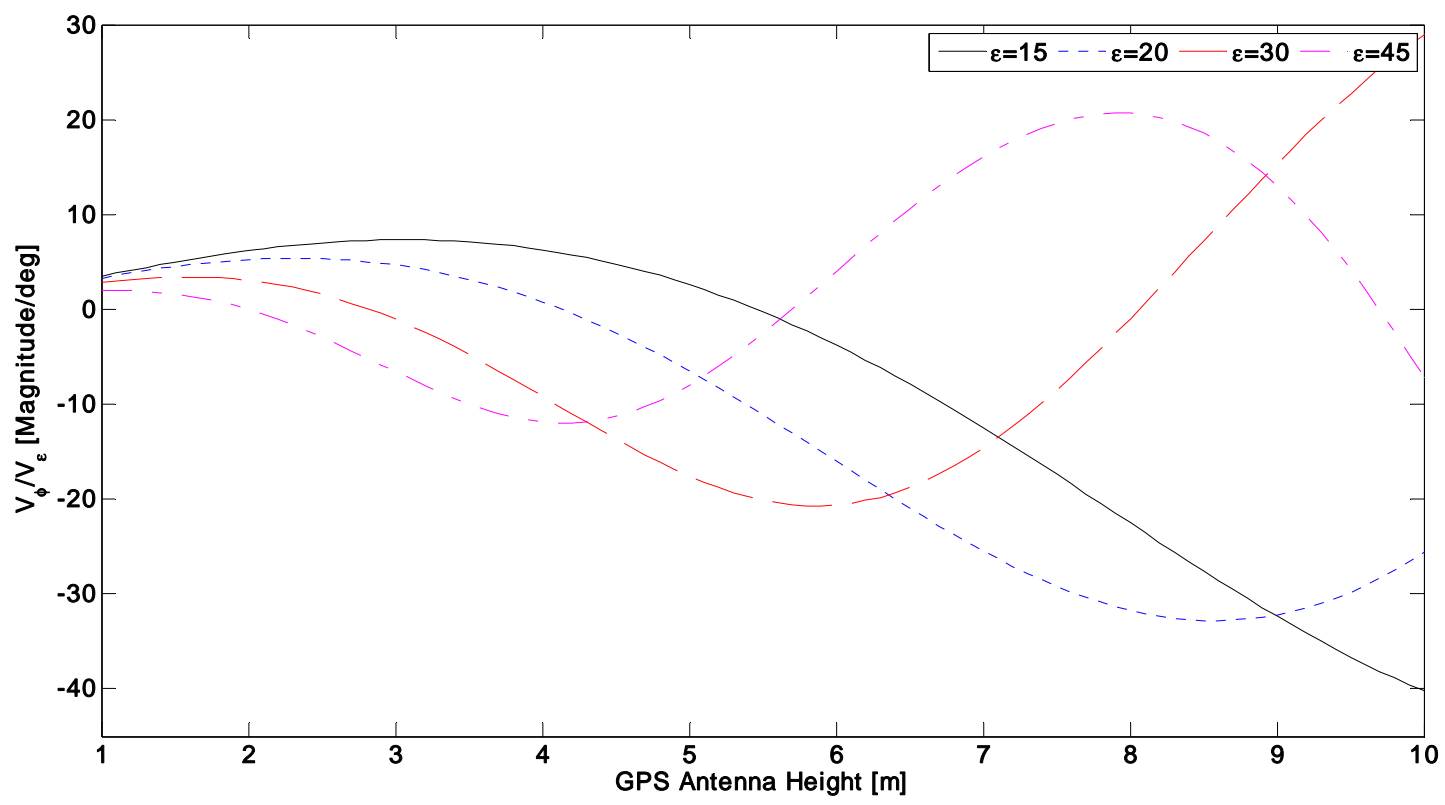


Although in this case where $H$ is constant and $\varepsilon$ is varying as GPS satellites are moving perpetually during the specified time span, but as presented in Figures 5 and 6 , the variability of multipath signature for $\varepsilon$ variations is more sensitive than $H$ ones. The $V_{\Phi} / V_{\varepsilon}$ for a determined $\mathrm{H}$ and $\varepsilon$ value has a much greater value than the same $H$ and $\varepsilon$ for $V_{\Phi} / V_{H}$ (for example in both Figures 5 and 6 , the unique values of $H=4 \mathrm{~m}$ and $\varepsilon=45^{\circ}$ have $V_{\Phi} / V_{H} \approx-3$ and $V_{\Phi} / V_{\varepsilon} \approx-10$ or $H=7 \mathrm{~m}$ and $\varepsilon=30^{\circ}$ have $V_{\Phi} / V_{H} \approx-1$ and $V_{\Phi} / V_{\varepsilon} \approx-20$, respectively). This demonstrates the determinant role of $\varepsilon$ in multipath signature variability when $\varepsilon$ is changing. According to this, an investigation is needed for the case where both $\varepsilon$ and $H$ are varied.

Therefore, to take into account the $\varepsilon$ variations during the certain time span, if $H$ changes sizably (e.g., due to snow accumulation or ice melting), it will lead to the sudden physical variations in ground GPS station's surrounds (Figure 7). By using Equations (5) and (10) as the derivatives of multipath signatures variations subjected to the variability in $H$ and $\varepsilon$, it can provide us with the trend of GPS scattered and reflected signals interacting with the ground GPS station receiver's environment, as shown in Figures 8 and 9, respectively.

Figure 7. GPS scattered and reflected signals towards the Earth's surface and from surrounding ground GPS station receiver during a specified time span, while $H$ is varying caused by snow accumulation (or snow and ice melting).

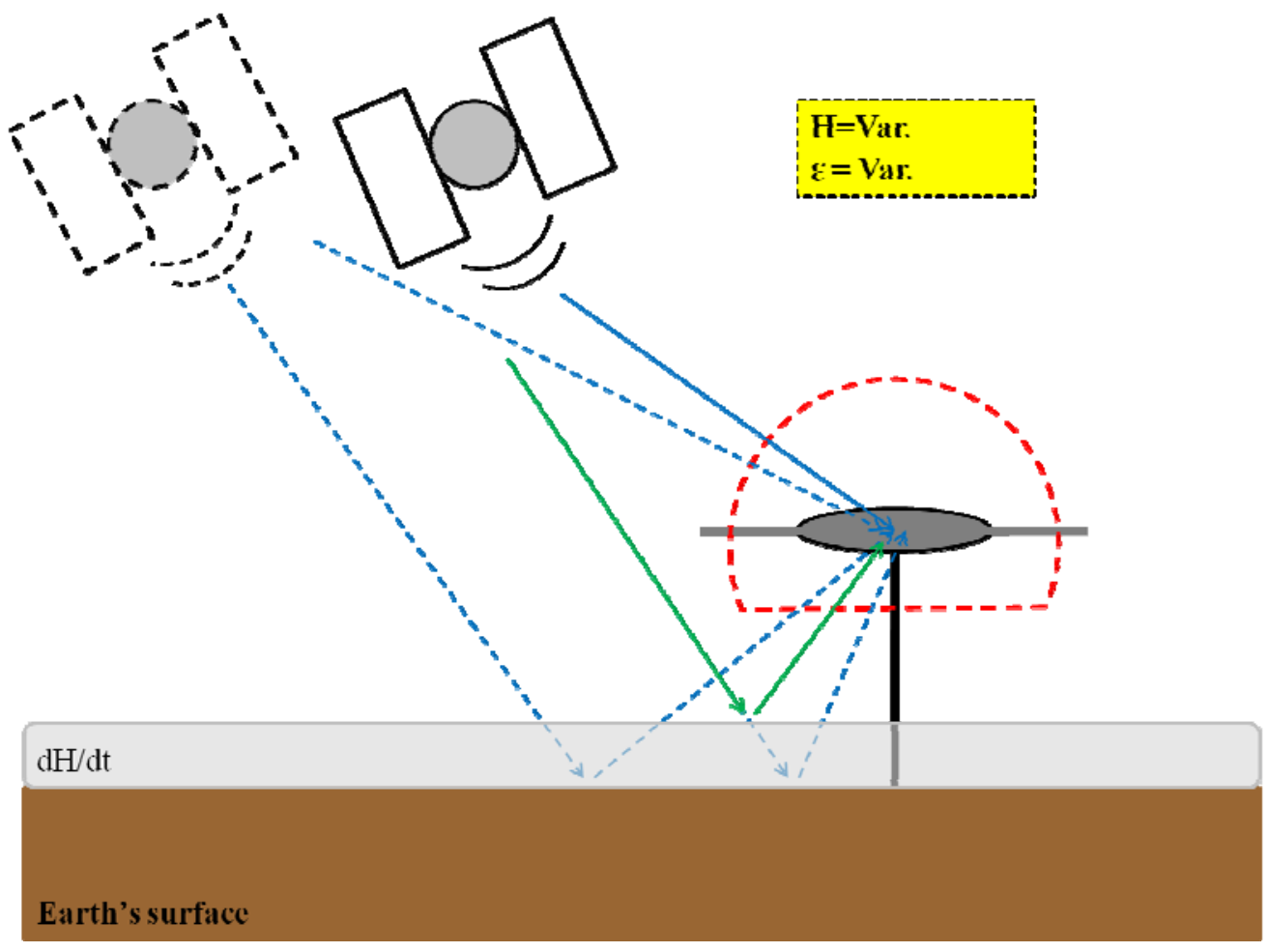

From Figure 7, when the GPS satellite location changes, obviously the scattered and reflected signals are changed as the direction of these signals is switching to different planes. However, as described in Figures 4-6, the $\varepsilon$ variations result in large multipath signature variability values; particularly a sudden small increase in $H$ can make it more sensitive to the small changes in $\varepsilon$, shown in both Figures 8 and 9. Additionally, the multipath signature variability with respect to $\varepsilon$ is more sensitive than $H$, which is clear by comparing Figure 8 and Figure 9. 
Similarly, if $\varepsilon$ does not vary during this time, or the ground GPS receiver only tracks a specific single GPS satellite, and in a certain elevation (e.g., Figure 10), the multipath signature variability values with respect to $H$ variations will be periodic. These variability values critically depend on $H$ (clearly, also depend on a fixed $\varepsilon$ value), which is demonstrated in Figure 11 for $H$ between a height of 8 and $8.8 \mathrm{~m}$.

The only reason that can restrict the reflected signal's angle when $\varepsilon$ is constant and $H$ is varied during the time is the sudden variation in $H$ (it may be due to $\mathrm{H}$ variation manually or changes in the physical conditions surrounding the ground receiver). As it is shown in Figure 11, a nearly $15 \mathrm{~cm}$ increase in $H$ value (e.g., from 8.15 to $8.3 \mathrm{~m}$ ) can lead to a significant change in multipath signature variability from a maximum value to a minimum one (See Figure 11). In addition, this variability has periodic trend and depends on a fixed $\varepsilon$ value which is considered here equal to $25^{\circ}\left(\varepsilon=25^{\circ}\right)$.

Figure 8. Variability of multipath signatures subjected to the changes in $H\left(V_{\Phi} / V_{H}\right)$ based on $H$ variations.

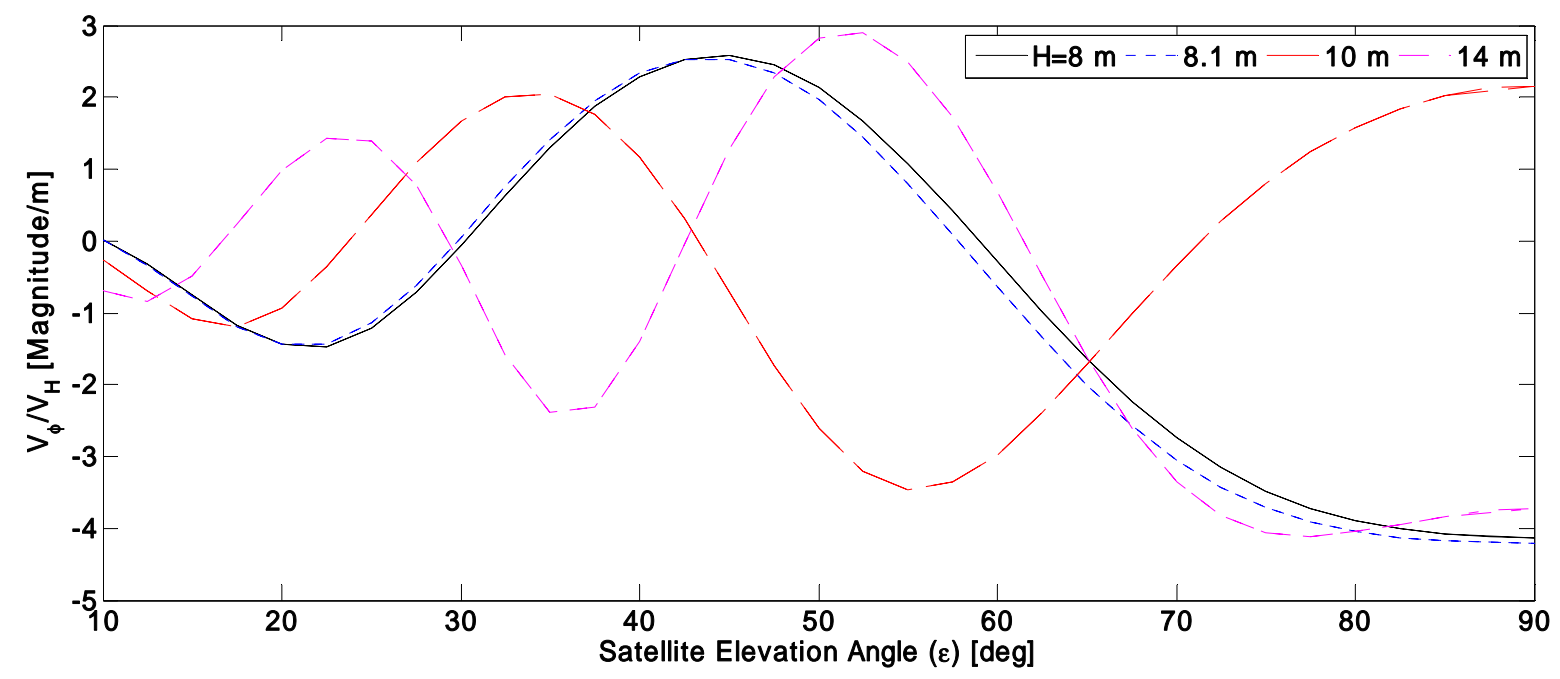

Figure 9. Variability of multipath signatures subjected to the changes in $\varepsilon\left(V_{\Phi} / V_{\varepsilon}\right)$ based on $H$ variations.

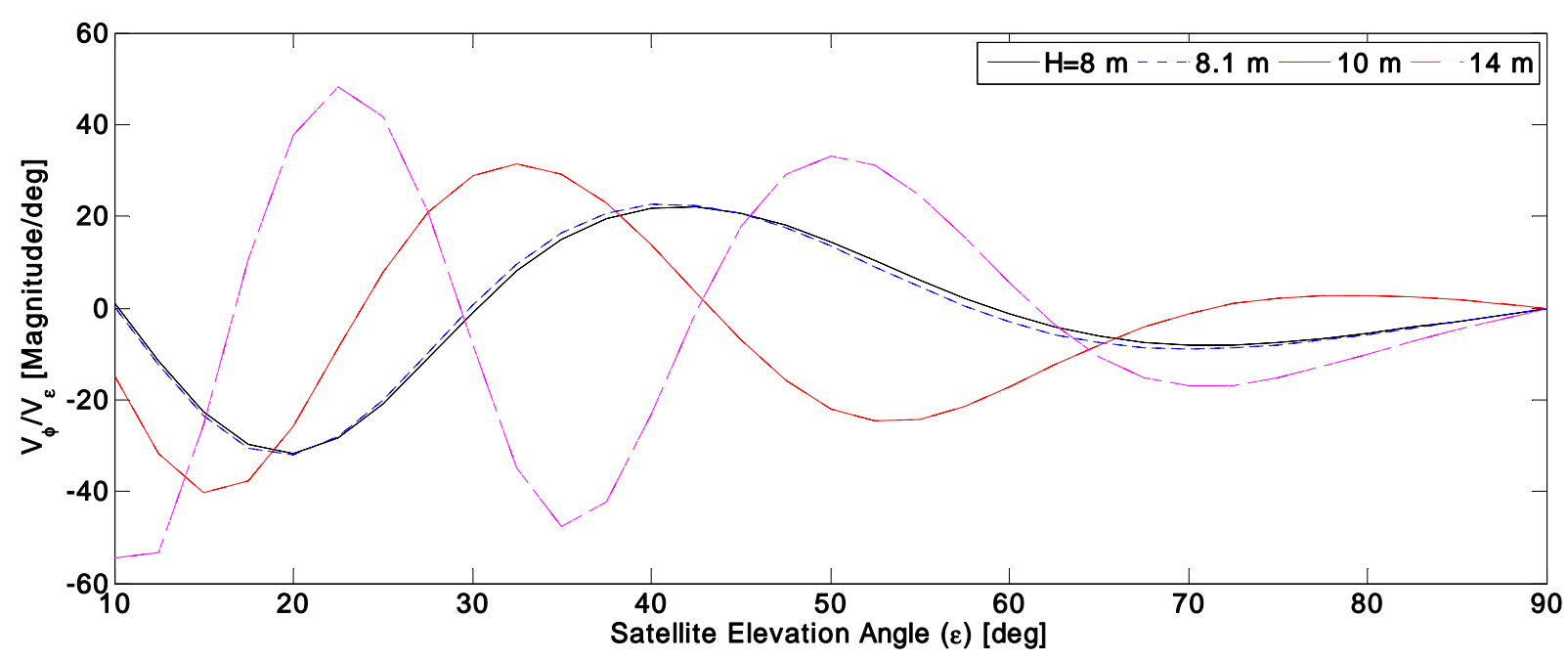


Figure 10. GPS scattered and reflected signal towards the Earth's surface and from surrounding ground GPS station receiver during a specified time span, while $\mathrm{H}$ is varying due to snow accumulation (or snow and ice melting) but the $\varepsilon$ is constant (here $\varepsilon=25^{\circ}$ ).

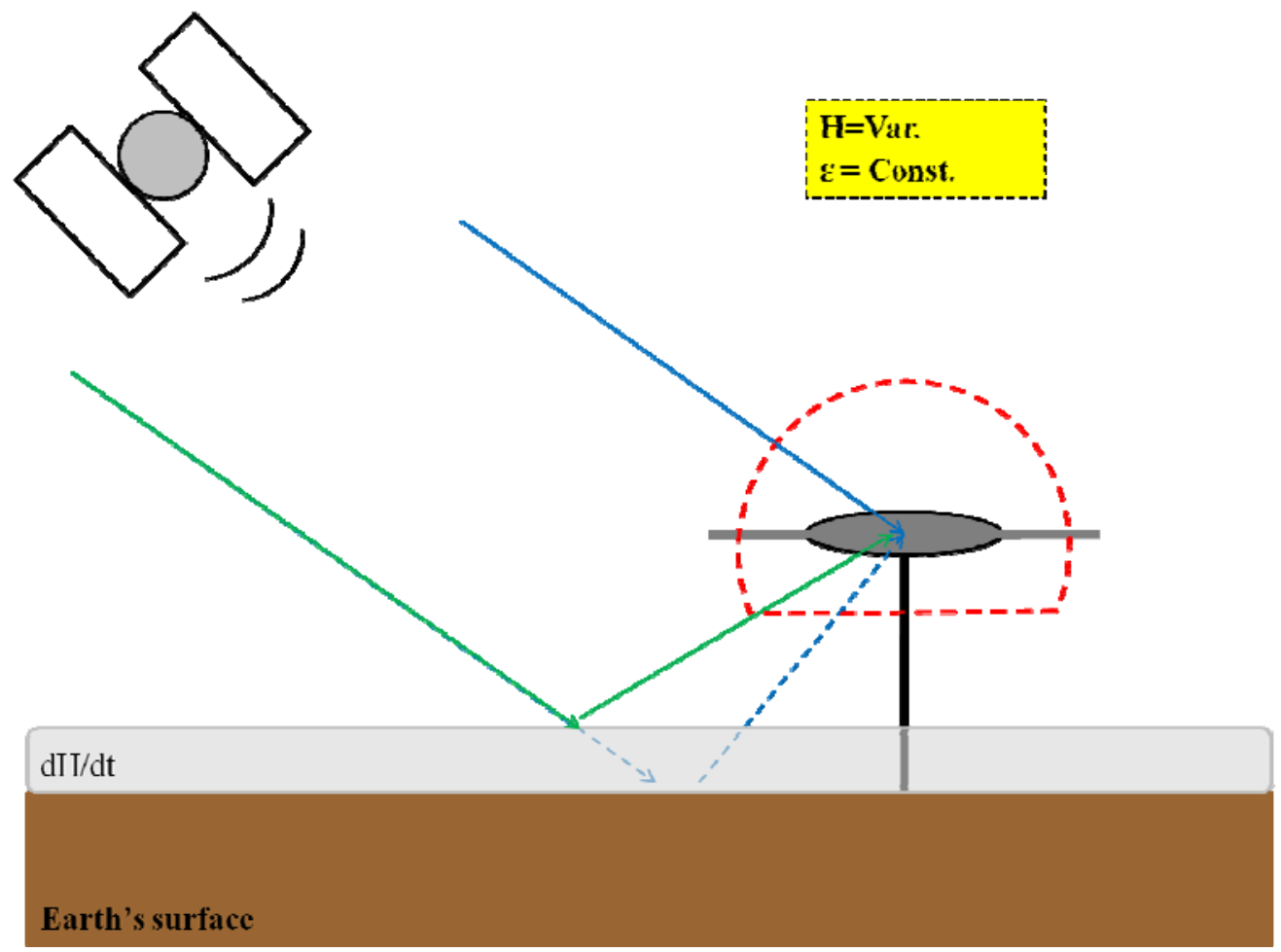

Figure 11. Variability of multipath signatures subjected to the changes in $H\left(V_{\Phi} / V_{H}\right)$ and fixed $\varepsilon$ (here $\varepsilon=25^{\circ}$ ) based on $H$ variations.

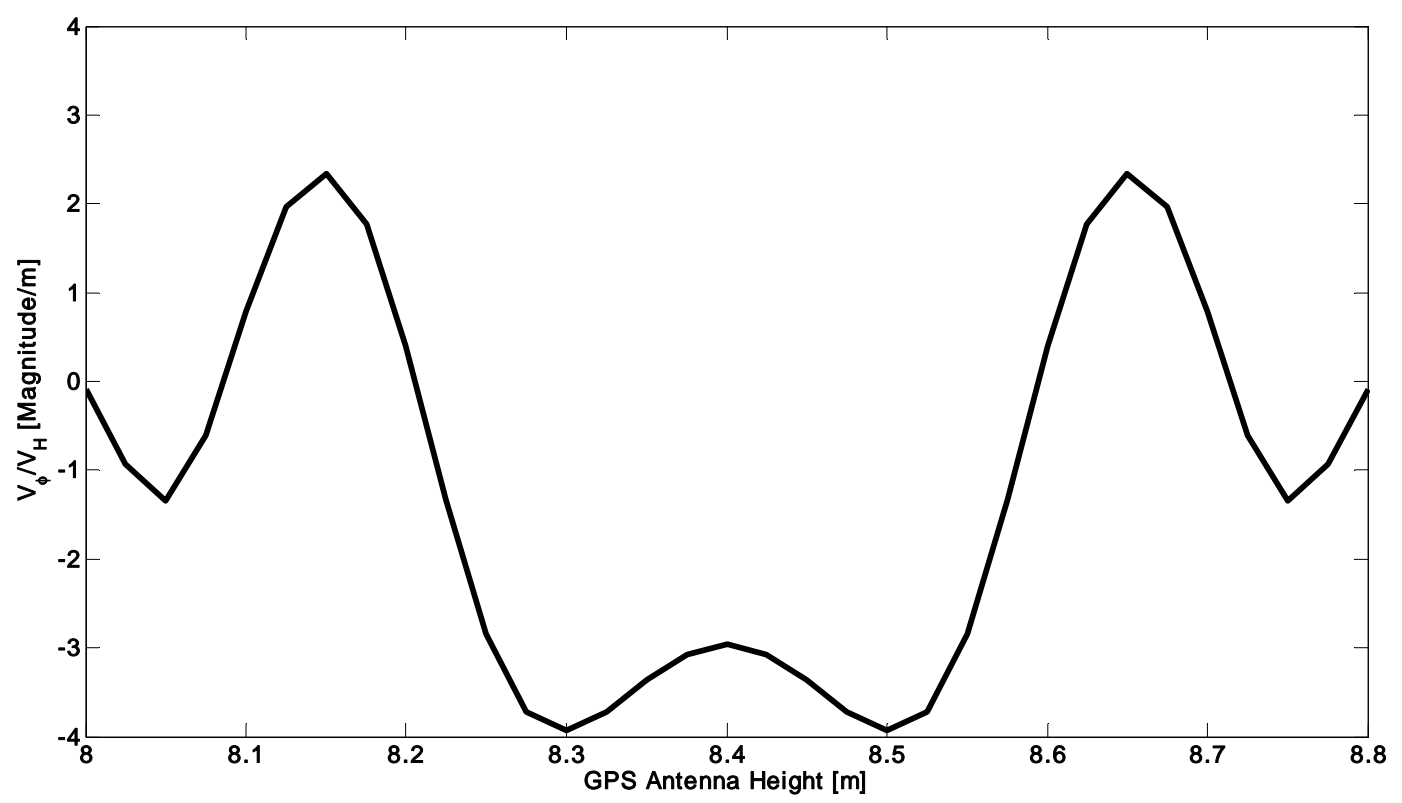

According to Equation (11), the multipath signature variability with respect to $H$ variations is directly a function of $\varepsilon$. Therefore, if we assume the following schematics for the GPS satellite and ground receiver, as shown in Figures 12 and 13. 
Figure 12. GPS scattered and reflected signal towards the Earth's surface and the from surrounds of the ground GPS station receiver during a specified time span while $H$ is the maximum and $\varepsilon$ is changing up to the satellite zenith angle (practically, this case may happen rarely).

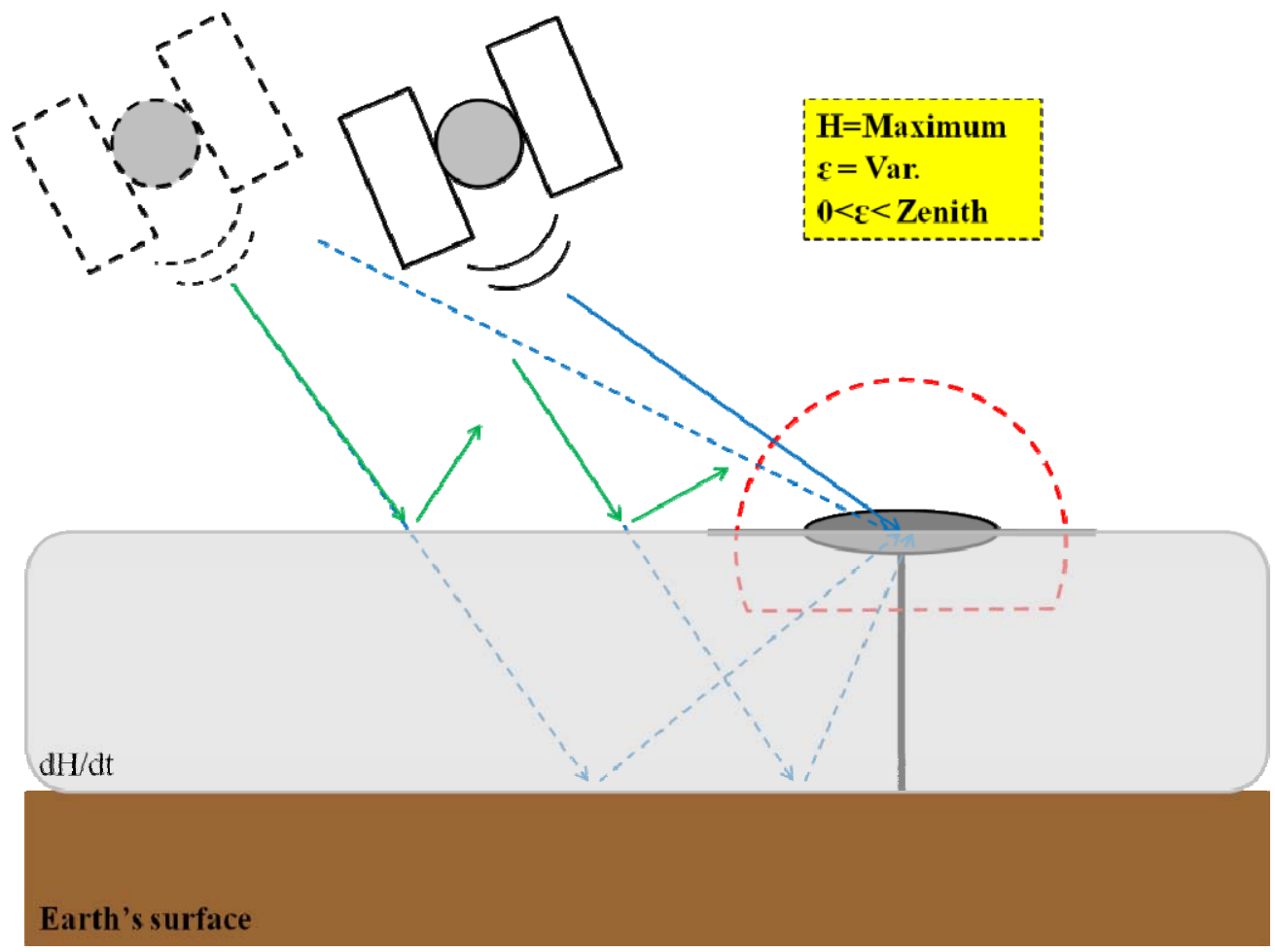

Figure 13. GPS scattered and reflected signal towards the Earth's surface and from the surrounds of the ground GPS station receiver during a specified time span while $H=0$ and $\varepsilon$ is changing up to the satellite zenith angle (practically, this case would not occur).

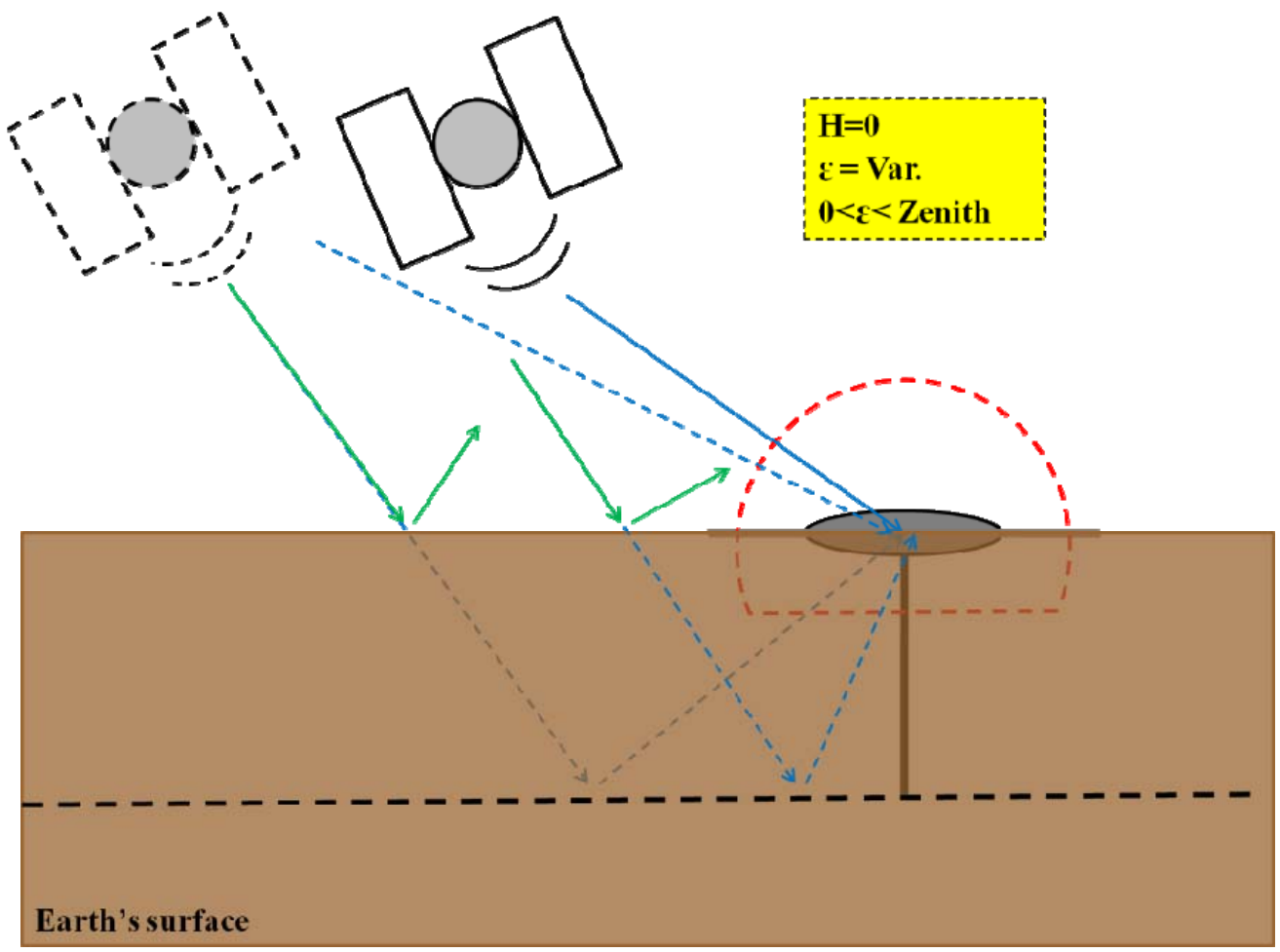


It is clear that the reflected signals from maximum snow surface (or ice surface) in Figure 12 or Earth's surface, represented in Figure 13, are similar in terms of reflected signal angle from snow (ice) and Earth's ground surface, respectively. However, there are differences in signal strength and amplitude as they have been reflected from different surfaces with unequal reflectance polarization coefficients, but as mentioned in the physical reflectivity's introduction, we concentrate only on the physical status of scattered and reflected signals interacting with the selected surfaces and ground receiver rather than signal strength and amplitude values. Therefore, the multipath signature variability subject to $H$ variations for both Figure $12(H=$ Maximum) and Figure $13(H=0)$ are similar. These different values for $\varepsilon$ with $H=0$, are presented in Figure 14.

Figure 14. Variability of multipath signatures subjected to $H$ variations $\left(V_{\Phi} / V_{H}\right)$ in $H=0$ for different $\varepsilon$ values.

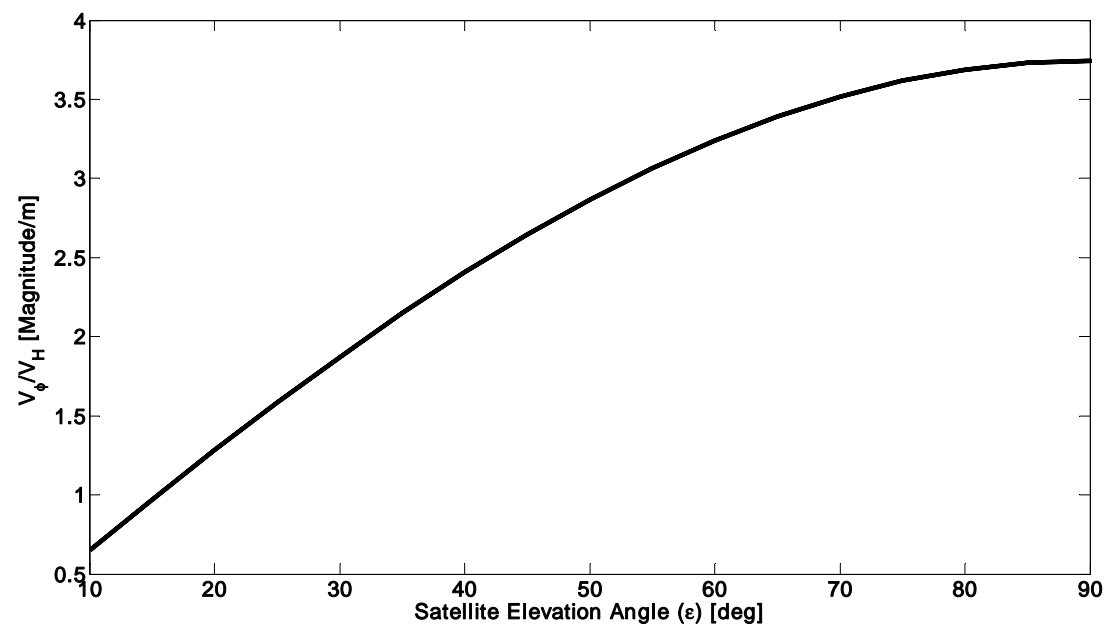

As it can be understood from Figures 12-14, increases in the satellite elevation angle up to its zenith angle lead to maximum variability in multipath signatures as the reflected signals are going to interact with the ground GPS receiver antenna. Moreover, the low satellite elevation angle gives more reflected signals from the surface rather than what can be received by the ground GPS receiver itself.

\subsection{GPS Reflected Signals Surface and Reflectance Characteristics}

The reflected signals from a surface are associated principally by two factors: the physical formation of interacting signals and the surface characteristic. The former is defined here as physical reflectivity of multipath signatures and the latter is described as reflectance characteristics. The physical reflectivity was expatiated completely in Section 3.1. In this part, the specular reflection and the total reflectivity as well as linear and circular polarization and their corresponding convolution functions are discussed in detail.

Theoretically, almost all objects can emit specular reflections, although the amount of the reflections varies and depends on the object's optical properties, such as the surface roughness, the Fresnel reflection coefficients, and so on [26]. Figure 15 presents a diagram of characteristic reflectance in interacting with incidence signal (scattered signal) from a certain surface. The result of such interaction is refracted and reflected signals with different surface polarization reflectances. 
Figure 15. Incident, reflected, refracted and Brewster angle and polarization of signal that occurs on an interface of a surface section at Brewster's angle (not to scale).

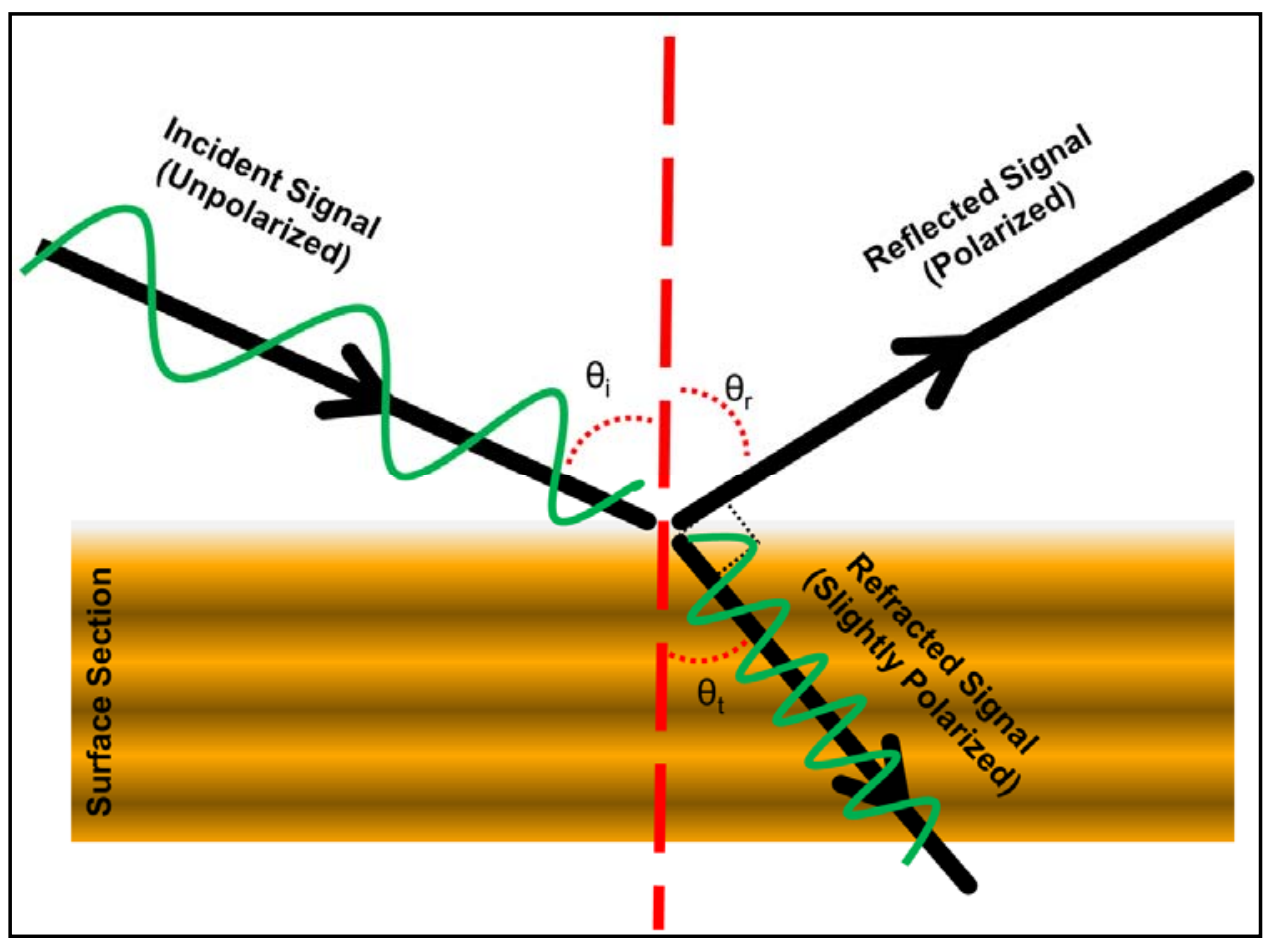

As pointed out in the theory and methodology section, the environment geometry of signals (incident and reflected signal angles) and the surface electrical properties (conductivity and relative permittivity) are playing consequential roles in the surface reflectance features where the reflection coefficients for a surface provide applicable information on the nature of reflected signals [27].

Figure 16. Perpendicular and parallel specular reflection coefficients for the main phases of the Earth's surface covered materials; frozen water (blue) and soil (red).

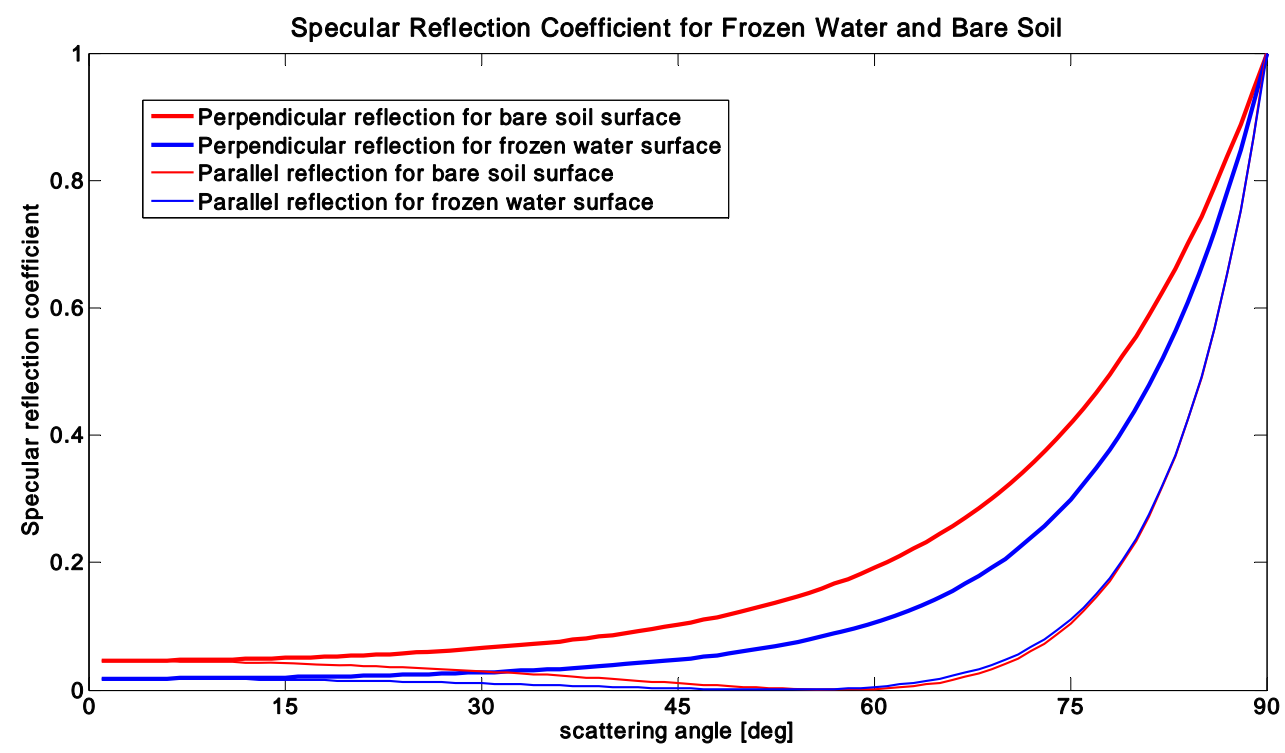

To consider the most significant phases of natural substances covered on the Earth's surface, frozen water (e.g., snow or ice) and soil (mixed with rock) are the most crucial surfaces with a refractivity 
index of 1.309 and 1.540, respectively [19], which have been analyzed through specular reflection coefficients versus scattering perpendicular and parallel angles (see Table 1). Figure 16 represents specular reflection coefficients for each selected surface and the parallel reflection coefficients with deceleration to reach a unique value, rather than perpendicular ones.

The specular reflection coefficients for each surface are increasing continuously and monotonically in Figure 16. Since the soil surface has maximum perpendicular reflection coefficients, the parallel specular reflection coefficients are almost in the same trend-value for both these two surfaces. Although each surface may change its reflection coefficients to signal scattering depending on the geometry or other significant environmental factors that eventually would affect these values, initially we assumed idealistic situations that are not changing for the scattered and reflected signals during these results.

In order to understand the entire frozen water and soil surface interaction for scattered signals, the total reflection of both perpendicular and parallel specular reflection is proposed. The total surface reflectivity is computing the half plane scattered angles (Figure 17), which shows a sudden phase change of $90^{\circ}$ for the selected surfaces. This sudden phase change happens when the total specular reflection coefficients increase up to 4 ; while the scattering angle approaches $180^{\circ}$. The bare soil is reflecting the incident signals more than frozen water for the entire scattering angles. In other words, frozen water absorbs more scattered signals than bare soil.

The comparison between bare soil and frozen water in terms of specular reflection and total reflection coefficients (Figures 16 and 17) shows that the frozen water's reflection is approximately equal to half the bare soil's reflection in interacting with incident signals for all scattered angles.

Figure 17. Total specular reflection coefficient based on both perpendicular and parallel specular reflection coefficients of two main phases of the Earth's surface covered materials; frozen water (blue) and soil (red) surface.

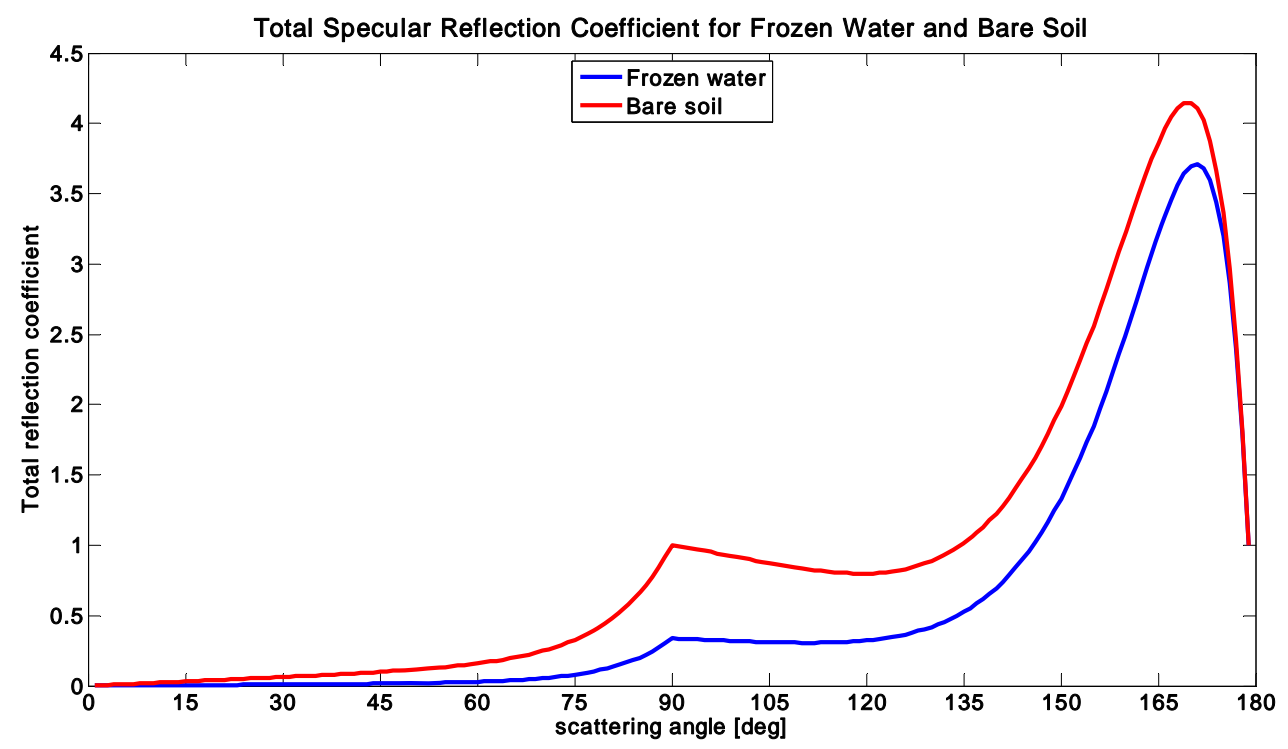

Originally, the GPS scattered signals are Right Handled Circulated Polarization (RHCP). The circular polarization occurred in the reflected surface is the vector sum of the linearly polarized waves (both in vertical and horizontal). Therefore, it is appropriate to consider the reflection coefficients for 
linear cases initially. In order to compute linear polarization reflection coefficients, the conductivity and relative permittivity values for selected surfaces including soil, snow and ice (as given in Table 1) and the equations for reflection coefficients in horizontal and vertical polarization (Equations (13) and (14)) are used. The linear polarization reflection coefficients for GPS signals ( $\mathrm{L} 1=1.575 \mathrm{GHz}, \lambda 1=19$ $\mathrm{cm}$ and $\mathrm{L} 2=1.227 \mathrm{GHz}, \lambda 2=24.4 \mathrm{~cm}$ ) are represented in Figure 18.

Figure 18. Linear polarization reflection coefficients vertically (red colored plot) and horizontally (blue colored plot) in interacting with GPS L1 (Top) and L2 (Down) signals for selected surfaces including snow, ice and soil.
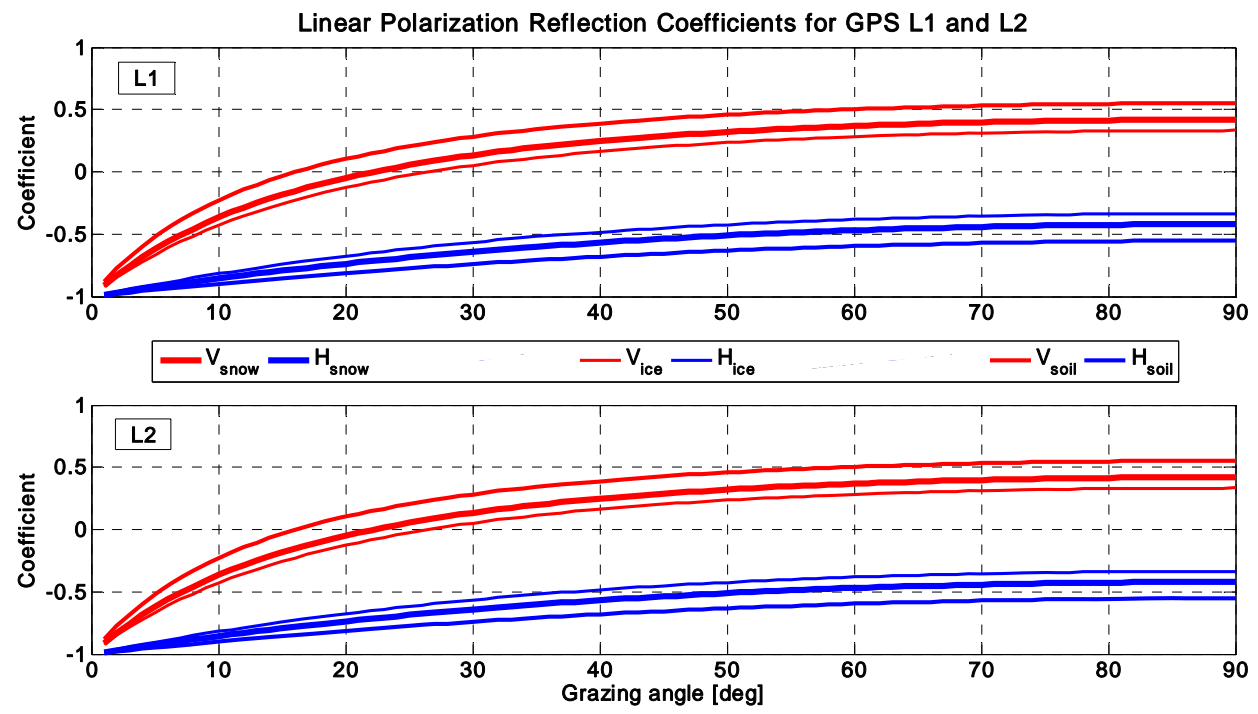

From Figure 18, it can be seen that the reflection coefficient values for each surface is varied and unique in comparison to the other surface. The vertical component (red colored plot) increases quickly compared to the horizontal one (blue colored plot) which increases gradually. Although the vertical reflection coefficient increases with approximate near zero slopes for the grazing angles that is greater than the Brewster angles, the linear polarization has a positive constant slope for the horizontal polarization.

In addition, the soil surface linear polarization coefficient for both GPS L1 and L2 signals has a maximum value in vertical polarization and the minimum value in horizontal polarization. Moreover, the ice surface linear polarization coefficient has a maximum value in horizontal polarization for both GPS signals. The snow surface has a lesser linear polarization value than soil in vertical polarization, but it has a greater value than soil in horizontal polarization when the incident signals are gradually getting linearly polarized by interacting with the surface.

Generally, for the angles less than the Brewster angles, the reflected signal, which is Right Hand Elliptically Polarized (RHEP), would become increasingly more elliptic as the Brewster angle is approaching [7]. Though at the Brewster angle, the polarization approaches that absolute point, which is Left Hand Elliptically Polarized (LHEP), with an elliptical increase as grazing angles increase [13].

Regarding the GPS multipath signatures and reflectance characteristics, the GPS receiver antenna only can receive RHCP signals (in fact those LHCP signals which can be received by GPS receiver antenna are at a lower level and negligible). Then, it is necessary to assess the circular polarization 
reflectance characteristics in the mentioned surfaces which the corresponding optical polarization approaches presented in Equations (15) and (16). The linear polarization is necessary to understand circular polarization as it is used in those related circular equations.

Referring to Figure 19, the co-polarized and cross-polarized plots are approximately symmetrical. The Brewster angle in circular polarization occurs at the intersection point of co-polarization and cross-polarization plots (e.g., $27^{\circ}$ for snow in GPS L1 signal).

Figure 19. Circular polarization as reflection coefficients of co-polarization (red) and cross-polarization (blue) in interacting with GPS L1 (Top) and L2 (Bottom) signals for selected surfaces including snow, ice and soil.
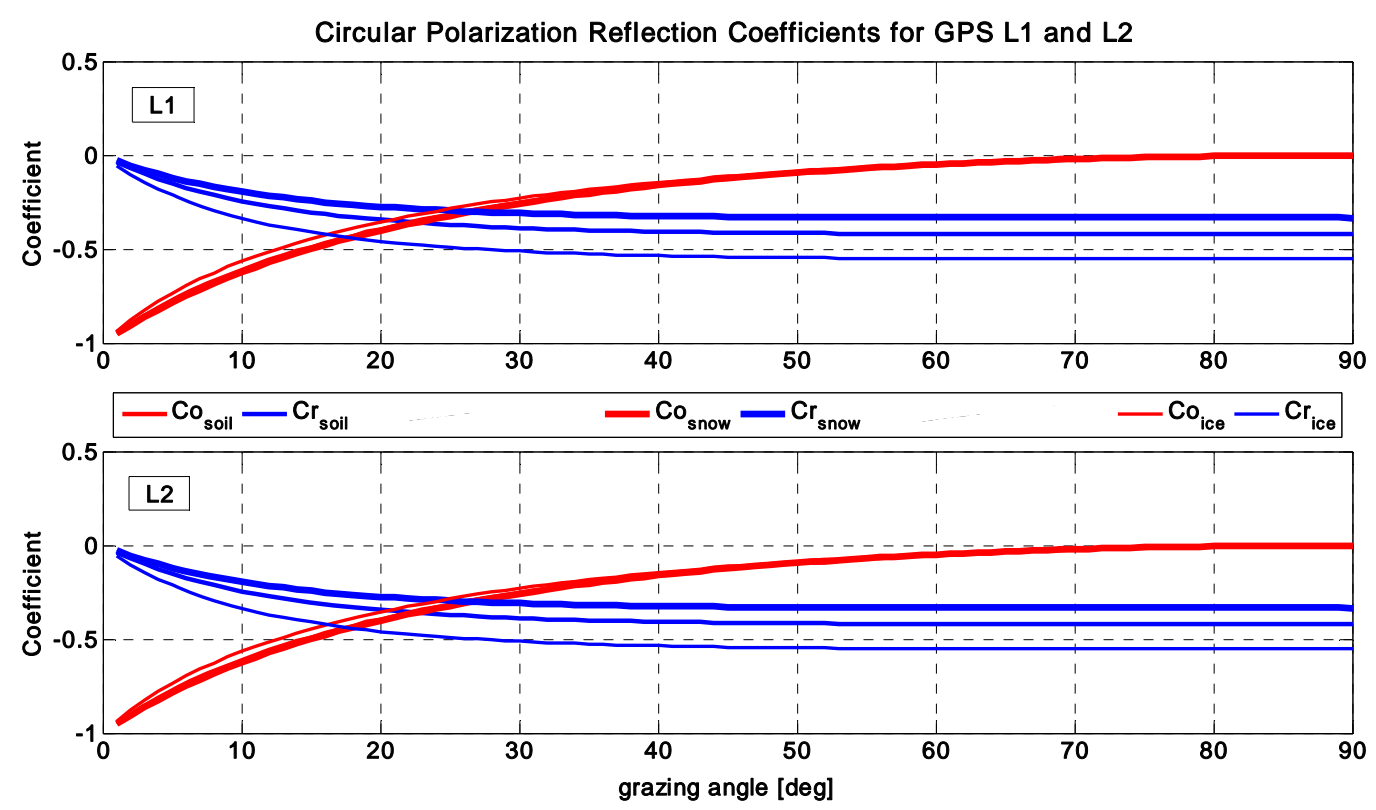

Additionally, ice surface reflectance has a minimum Brewster angle, but snow surface has a maximum one. The soil surface's Brewster angle is less than the snow's one but larger than soil surface's value. This can be attributed to the large difference between snow and ice surface in terms of characteristic reflectance discriminating in interactions with GPS signals in circular polarization.

To demonstrate this, by referring to Figure 18 (linear polarization plots for GPS L1 and L2 signals) and Equations (15) and (16) that consist of linear polarization values, the difference between vertical and horizontal plots can be seen as the largest values in soil surface plots.

In order to observe the entire reflection from these surfaces (both of vertical and horizontal polarizations or both of co-polarization and cross-polarization reflections) the convolution functions have been proposed for soil, snow and ice surface in both linear (Equation (17)) and circular (Equation (18)) polarization, respectively(Figures 20 and 21).

In Figure 20, the minimum of each surface occurs at $90^{\circ}$. This is where the first derivative of the corresponding convolution function for the linear polarization can occur. This means the behavior of the convolution function is inverting suddenly after $90^{\circ}$. In other words, this sudden reversing is similar in order to replace the vertical linear polarization by a horizontal one after $90^{\circ}$. Furthermore, it can be concluded from each convolution values' diagram that each selected surface has a majority of negative linear polarization values rather than positive ones. 
Figure 20. Linear polarization convolution values for GPS L1 (Top) and L2 (Bottom) signals interacted to selected surfaces including snow, ice and soil.
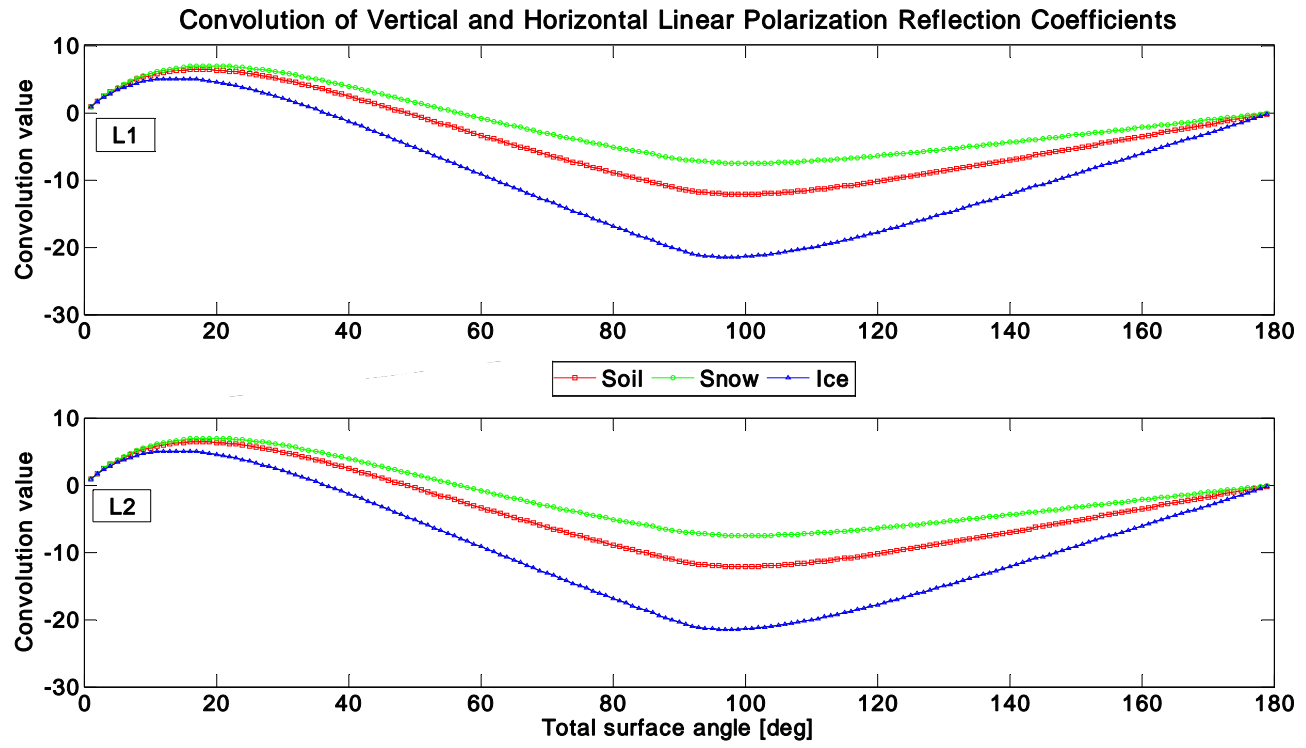

Figure 21. Circular polarization convolution values for GPS L1 (Top) and L2 (Down) signals interacted to selected surfaces including snow, ice and soil.
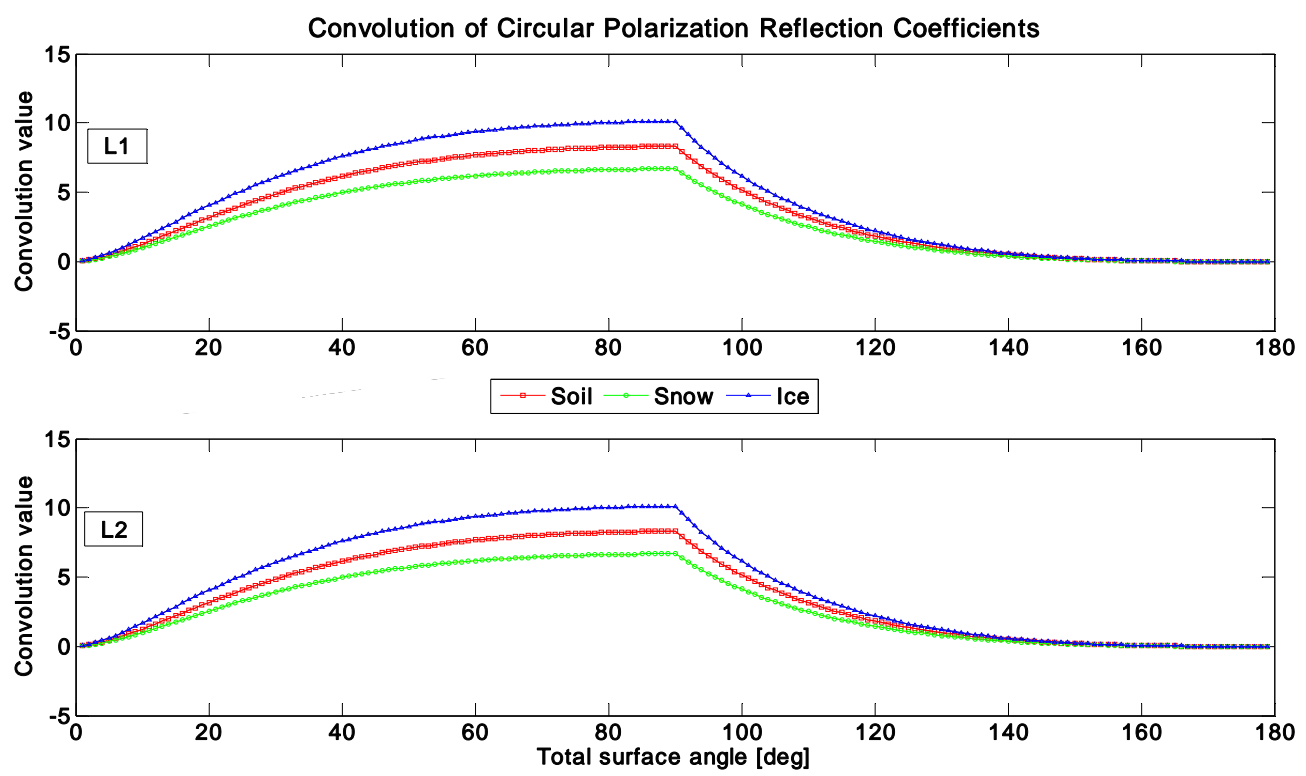

Since it is presented in Figure 20, the soil surface total reflection convolution values derived from linear polarization is located between ice and snow values. This is highly remarkable and can be strong evidence for discriminating snow and ice surfaces from each other through GPS reflected signals.

The comparison of linear polarization convolution values for GPS L1 and L2 signals (see Figure 20) gives us the fact that the surface reflectance for GPS L2 signal is larger than L1; although the GPS L2's wavelength is greater than L1.

Referring to Figure 18 and linear polarization equations in GPS L1 and L2 signals for soil, the difference between vertical and horizontal (blue and red colored plots) is more than other surfaces. For example, in grazing angle of $30^{\circ}$ for GPS L1 signal; the linear polarization reflection coefficient for 
soil, snow and ice surfaces in vertical and horizontal polarizations are as follows, respectively: 0.28 , $0.21,0.09$ and $-0.74,-0.62,-0.55$ (see Figure 18 ). The absolute difference between each pair value for soil, snow and ice surfaces will be equal to $1.02,0.83$ and 0.64 . Therefore, the summation of soil surface reflection coefficients in vertical and horizontal directions computed by convolution function is displayed as more noteworthy.

The co-polarized and cross-polarized coefficient values vary between 0 and -1 (see Figure 19). In Figure 21, the changing phase angle occurs at $90^{\circ}$. The circular convolution function trend before and after $90^{\circ}$ is becoming completely shifted and mirrored with a $180^{\circ}$ phase difference. Moreover, the point which $R C_{H}$ and $R C_{V}$ are equivalent to each other is occurring at $0^{\circ}$ where the cross-polarization coefficient reflectance is equal to 0 . In addition, the summation of $R C_{H}$ and $R C_{V} \mathrm{~s}$ equal to 0 where the co-polarization reflectance coefficient is equal to zero at $90^{\circ}$.

Additionally, the snow surface has the minimum circular reflection value in co-polarization and maximum one in cross-polarization. However, the ice surface has maximum circular reflection values in co-polarization and minimum values in cross-polarization. In fact, referring to Equations (15) and (16) and Figures 19 and 21, the Brewster angle for snow surface is greater than soil surface in cross-polarized and co-polarized GPS L1 and L2 signals. Similarly, the Brewster angle for soil surface is larger than ice surface values.

To compare these two convolution functions for linear (Figure 20) and circular polarization (Figure 21), for both of them the sequence order for surface reflectance in GPS L1 and L2 signals is as follows: the total snow surface's reflectance values are larger than both soil and ice surface's reflectance values during linear polarization. In circular polarization, the total reflectance of cross-polarization and co-polarization for ice surface is greater than both soil and ice surface. For example, for GPS L1 signal in total surface angle at $40^{\circ}$, the linear polarization convolution and circular polarization convolution values for soil, snow and ice surfaces are respectively as follows: 4 , $6,-2$ and $6,4,8.5$. The soil surface reflectance values in both linear and circular polarization convolution functions are located between ice and snow surface's reflectance values, as shown in Figures 20 and 21.

\section{Conclusions}

The modeled surfaces including soil, snow and ice have special physical reflectivity and reflectance characteristics in interacting with GPS L1 and L2 signals. It is necessary to consider both physical reflectivity and reflectance characteristics when we deal with GPS-Reflectometry applications, such as snow depth or height and ice thickness measurements. Since in physical reflectivity, the GPS antenna height and satellite elevation angle have maximum effect on variability of GPS reflected signals, the role of satellite elevation angle variations on multipath variability is more significant especially when the physical surface formation surrounding the ground GPS station receiver is changing during the certain time span. In fact, the variability of multipath signature depends critically on satellite elevation angle variations rather than the GPS antenna height, while the GPS antenna height variations are being caused mostly by snow accumulation or ice melting, particularly in snow-covered ground and permafrost at high latitudes. 
In regards to surface reflectance characteristics, the Brewster angle is the intersection point of linear vertical and horizontal polarization plots. Since GPS antenna receives RHCP signals mostly, the co-polarized signal in circular polarization is analogous to RHCP polarization. Additionally, in polarization convolution functions and integrals, the phase change point occurs at 90 degrees. This is where the general trend before and after 90 degrees is completely, symmetrically mirrored. The soil surface reflectance values are located between snow surface and ice surface ones (see Figures 20 and 21). Accordingly, the different surface reflectance values in linear polarization and circular polarization (co-polarization and cross-polarization) between snow surface and ice surface are noteworthy and can be computed precisely (for both GPS L1 and L2 signals). Therefore, in cryosphere studies such as snow depth and ice thickness measurements using GPS-Reflectometry technique, the different surface reflectance values for linear and circular polarizations among soil, snow and ice play a critical role in discriminating soil, snow and ice surfaces from each other. This discrimination between surfaces can be improved by considering the physical reflectivity of GPS scattered and reflected signals based on satellite elevation angle and GPS antenna height variations (see Figures 4-9).

In the future, further studies and practical case tests related to the surface physical reflectivity and polarization characteristics are needed in order to better understand the behavior of the targeted surfaces in interacting with GPS signals. This can be assessed by considering also the complicated conditions, such as the slope of the surfaces and the density variability of scattered and reflected signals for different GPS receivers.

\section{Acknowledgments}

The authors are greatly thankful to the reviewers and editors for their constructive comments and useful suggestions to improve the manuscript. This research is supported by Main Direction Project of Chinese Academy of Sciences (Grant No. KJCX2-EW-T03), Shanghai Science and Technology Commission Project (Grant No. 12DZ2273300), Shanghai Pujiang Talent Program Project (Grant No. 11PJ1411500) and National Natural Science Foundation of China (NSFC) Project (Grant No. 11173050).

\section{Conflict of Interest}

The authors declare no conflict of interest.

\section{References}

1. Jin, S.G.; Komjathy, A. GNSS reflectometry and remote sensing: New objectives and results. Adv. Space Res. 2010, 46, 111-117.

2. Jin, S.G.; Feng, G.; Gleason, S. Remote sensing using GNSS signals: Current status and future directions. Adv. Space Res. 2011, 47, 1645-1653.

3. Chappell, A.; Zobeck, T.M.; Brunner, G. Using bi-directional soil spectral reflectance to model soil surface changes induced by rainfall and wind-tunnel abrasion. Remote Sens. Environ. 2006, 102, 328-343.

4. Xu, D.Q.; Ni, G.Q.; Jiang, L.L.; Shen, Y.T.; Li, T.; Ge, S.L.; Shu, X.B. Exploring for natural gas using reflectance spectra of surface soils. J. Adv. Space Res. 2008, 41, 1800-1817. 
5. Maignan, F.; Breon, F.M.; Fedele, E.; Bouvier, M. Polarized reflectances of natural surfaces: Spaceborne measurements and analytical modeling. Remote Sens. Environ. 2009, 113, 2642-2650.

6. Helm, A.; Stosius, R.; Montenbruck, O.; Asjaee, J.; Yudanov, S.; Beyerle, G.; Rothacher, M. The GITEWS GNSS Occultation, Reflectometry and Scatterometry Space Receiver GORS. In Proceedings of GITEWS Workshop on New Earth Observation Techniques for Tsunami Detection and Geohazard Monitoring, Jakarta, Indonesia, 26-28 November 2007.

7. Hannah, B.M. Modeling and Simulation of GPS Multipath Propagation. Ph.D. Thesis, the Cooperative Centre for Satellite Systems, Queensland University of Technology, Kelvin Grove, QLD, Australia, 2001.

8. Litvinov, P.; Hasekamp, O.; Dubovik, O.; Cairns, B. Model for land surface reflectance treatment: Physical derivation, application for bare soil and evaluation on airborne and satellite measurements. J. Quant. Spectrosc. Radiat. 2012, 113, 2023-2039.

9. Chew, C.C.; Small, E.E.; Larson, K.M.; Zavorotny, V.U. Effects of near-surface soil moisture on GPS SNR data: Development of a retrieval algorithm for soil moisture. IEEE Trans. Geosci. Remote Sens. 2013, 9, 1-7.

10. Elosegui, P.; Davis, J.L.; Jaldehag, R.K.; Johansson, J.M.; Niell, A.E.; Shapiro, B. Geodesy using the global positioning system: The effects of signal scattering. J. Geophys. Res. 1995, 100, 9921-9934.

11. Ray, J.K.; Cannon, M.E. Mitigation of static carrier-phase multipath effects using multiple closely spaced antennas. Navigation 1999, 46, 193-201.

12. Bakshi, U.A.; Bakshi, A.V. Unit 3: Electric and Magnetic Fields in Materials. In Electromagnetic Fields; Technical Publications Pune: Pune, India, 2010; pp. 3-70.

13. Mott, H. Representation of Wave Polarization. In Polarization in Antennas and Radar, 1st ed.; Wiley-Interscience: New York, NY, USA, 1986; pp. 54-280.

14. Lakhtakia, A. General schema for the Brewster conditions. Optik 1992, 90, 184-186.

15. Hecht, E. Electromagnetic Properties of Light. In Optics, 4th ed.; Addison-Wesley: Boston, MA, USA, 2002; pp. 263-520.

16. Litvinov, P.; Hasekamp, O.; Cairns, B. Models for surface reflection of radiance and polarized radiance: Comparison with airborne multi-angle photopolarimetric measurements and implications for modeling top-of-atmosphere measurements. Remote Sens. Environ. 2011, 115, 781-792.

17. Kopp, M. Electrical conductivity of the snow (In French). J. Appl. Math. Phys. 1962, 13, 431-444.

18. Evans, S. Dielectric properties of ice and snow-A review. J. Glaciol. 1965, 5, 773-792.

19. ITU. Electrical Characteristics of the Surface of the Earth; Recommendation (ITU-Report) P.527-3; ITU: Geneva, Switzerland, 1992; pp. 1-5.

20. Van Nee, R.D.J. Multipath effects on GPS code phase measurements. Navigation 1992, 39, 177-190.

21. Kleusberg, A.; Teunissen, P.J.G. 3. Propagation of the GPS Signals. In GPS for Geodesy; Springer-Verlag: Berlin/Heidelberg, Germany, 1996; pp. 103-140.

22. Balvedi, G.C.; Walter, F. Analysis of GPS Signal Propagation in Tropospheric Ducts Using Numerical Methods. In Proceedings of 11th URSI Commission Open Symposium on Radio Wave Propagation and Remote Sensing Proceedings, Rio De Janeiro, RJ, Brazil, 30 October-2 November 2007. 
23. Vickerman, J.C.; Gilmore, I.S. Surface Structure Determination by Interference Techniques. In Surface Analysis - The Principal Techniques, 2nd ed.; John Wiley and Sons Ltd.: Chichester, UK, 2009; Chapter 8; pp. 391-478.

24. Leroux, C.; Deuze, J.L.; Goloub, P.; Sergent, C.; Fily, M. Ground measurements of the polarized bidirectional reflectance of snow in the near-infrared spectral domain: Comparisons with model results. J. Geophys. Res. 1998, 103, 19721-19731.

25. Bracewell, R. The Fourier Transform and its Applications, 2nd ed.; McGraw-Hill: New York, NY, USA, 1986; pp. 1-474.

26. Torrance, K.E.; Sparrow, E.M. Theory for off-specular reflection from roughened surfaces. J. Opt. Soc. Amer. 1967, 57, 1105-1112.

27. Salminena, M.; Pulliainen, J.; Metsamaki, S.; Kontu, A.; Suokanerva, H. The behavior of snow and snow-free surface reflectance in boreal forests: Implications to the performance of snow covered area monitoring. Remote Sens. Environ. 2009, 113, 907-918.

(C) 2013 by the authors; licensee MDPI, Basel, Switzerland. This article is an open access article distributed under the terms and conditions of the Creative Commons Attribution license (http://creativecommons.org/licenses/by/3.0/). 